\title{
On Uniform Asymptotic Stability of Time-Varying Parameterized Discrete-Time Cascades
}

\author{
Dragan Nešić and Antonio Loría, Member, IEEE
}

\begin{abstract}
Recently, a framework for controller design of sampled-data nonlinear systems via their approximate discrete-time models has been proposed in the literature. In this paper, we develop novel tools that can be used within this framework and that are useful for tracking problems. In particular, results for stability analysis of parameterized time-varying discrete-time cascaded systems are given. This class of models arises naturally when one uses an approximate discrete-time model to design a stabilizing or tracking controller for a sampled-data plant. While some of our results parallel their continuous-time counterparts, the stability properties that are considered, the conditions that are imposed, and the the proof techniques that are used, are tailored for approximate discrete-time systems and are technically different from those in the continuous-time context. A result on constructing strict Lyapunov functions from nonstrict ones that is of independent interest, is also presented. We illustrate the utility of our results in the case study of the tracking control of a mobile robot. This application is fairly illustrative of the technical differences and obstacles encountered in the analysis of discrete-time parameterized systems.
\end{abstract}

Index Terms-Casacded systems, discrete-time, Lyapunov stability, nonholonomic systems.

\section{INTRODUCTION}

$\mathbf{T}$ HE prevalence of digitally controlled systems and the fact that the nonlinearities in the plant model can often not be neglected, strongly motivate the area of nonlinear sampled-data systems. A typical nonlinear sampled-data system consists of a nonlinear continuous-time plant and a nonlinear discrete-time controller that are interconnected via the analog-to-digital (A-D) and digital-to-analog (D-A) converters.

Despite the importance of this class of systems, few systematic tools for nonlinear sampled-data controller design are available in the literature. One way of studying the control and analysis problems for sampled-data systems is the hybrid systems approach within which we may cite among others [4], [46], [21], [8], [9], [40]. Another control method for this class of systems is to discretize the plant model and use it for controller design. Most of such references using this approach assume existence of the exact discrete-time plant model (see, for instance, [49], [18], and [17]). While this approach is standard for linear systems, it is typically not straightforward

Manuscript received July 23, 2003; revised December 1, 2003. Recommended by Associate Editor Z.-P. Jiang. This work was supported by the Australian Research Council under the Large Grants Scheme.

D. Nešić is with the Department of Electrical and Electronic Engineering, The University of Melbourne, Parkville, 3010 VIC, Australia.

A. Loría is with the C.N.R.S, LSS—Supélec, Plateau de Moulon, 91192 Gif sur Yvette, France.

Digital Object Identifier 10.1109/TAC.2004.829645 for nonlinear systems. Indeed, it is usually not possible to discretize a nonlinear plant model exactly but only approximately using numerical methods such as Runge-Kutta and, hence, controller design has to be based on an approximate discrete-time model. Early results using this approach can be found in [5] and [6], and in the more recent works [28], [29], [25], and [1]. Moreover, it was shown in [34] that a controller may stabilize an approximate model while destabilizing the exact model for all sampling periods. For example, if we design a minimum-time dead-beat controller for the Euler approximate model of a triple integrator, the controller will stabilize the Euler mode for any sampling period but destabilize the exact model for all sampling periods. Recently, a framework for nonlinear sampled-data controller design via their approximate discrete-time models was proposed in [34]-[36]. Furthermore, in [35], it was shown that stability of the exact discrete-time model under mild conditions guarantees also stability of the sampled-data system. Hence, the results of [34] and [35] provide a framework for controller design of sampled-data nonlinear systems via their approximate discrete-time models. Several constructive methods for controller design within the above mentioned framework has been reported in the literature (see [36], [19], [7], and the references cited therein).

Within the aforementioned framework, we often need to check appropriate stability properties of an approximate closed loop system that is described by a parameterized (in the sampling period) discrete-time model (see [34, Ths. 1 and 2]). In particular, one needs to check semiglobal practical uniform asymptotic stability (SP-UAS, cf. Definition 1) of the approximate discrete-time closed loop system in order to conclude (under additional assumptions) the same property for the exact discrete-time closed-loop system. The results from [35] allow to conclude SP-UAS for the sampled-data control system from SP-UAS of the exact discrete-time system. In words, SP-UAS for a given system means that its trajectories starting from any compact set converge to a closed neighborhood of the origin and, moreover, the set of initial conditions may be enlarged and the attractive neighborhood diminished as the sampling period is reduced.

The SP-UAS property is typically hard to check. The results that we present in this paper facilitate checking SP-UAS in the special case when the model has a cascaded structure. In this case, we can use the approach of "divide and conquer" by decomposing the higher dimensional problem into several lower dimensional problems that are easier to solve. A large number of results and applications of continuous-time cascaded systems can be found in [3], [20], [22], and [39] and discrete-time cascaded systems in [12], [23], and the references cited therein. 
We present sufficient and necessary conditions for SP-UAS of parameterized discrete-time cascaded systems of the form

$$
\begin{aligned}
& x(k+1)=f_{T}(k, x(k), z(k)) \\
& z(k+1)=g_{T}(k, z(k))
\end{aligned}
$$

where $x \in \mathbb{R}^{n_{x}}, z \in \mathbb{R}^{n_{z}}$ and $T>0$ is a parameter (usually the sampling period). These models naturally arise when an approximate discrete-time model of sampled-data nonlinear system, such as the Euler model, is used for controller design (see [34], [36], and the case study in Section IV). We also make use of the auxiliary system

$$
x(k+1)=f_{T}(k, x(k), 0) .
$$

Our main results (Theorems 1 and 2) state that if the systems (2) and (3) are SP-UAS and the solutions of (1) and (2) are uniformly bounded, then the system (1) and (2) is SP-UAS. We emphasize that even in the context of continuous-time systems, boundedness of solutions of the perturbed system (1) is not obtained from the stability properties of (2) and (3). For instance, one may have that (3) is globally asymptotically stable and (2) is globally exponentially stable and the solutions of (1) are unbounded; see, for instance, [48]. In [23], we give sufficient conditions for uniform boundedness of the solutions of (1).

Theorems 1 and 2 use slightly different assumptions and definitions of stability. In particular, Theorem 1 uses "trajectory based" conditions whereas Theorem 2 uses "Lyapunov based" conditions that may be not equivalent in general. Two direct consequences of these results are Corollaries 1 and 2 for nonparameterized discrete-time systems that generalize the cascades results of [12]. We also present several sufficient conditions for our conditions to hold and most notably in Section III-B we construct strict Lyapunov functions from nonstrict ones (for similar constructions in continuous-time systems see [26]). Finally, through a case-study of the unicycle benchmark problem we illustrate how using our results we can obtain a new control algorithm for the unicycle that can be regarded as a redesign of the continuous-time controller in [37]. Moreover, our controller outperforms, in simulations, the continuous-time controller from [37] which is discretized and implemented using a sampler and zero order hold.

The paper is organized as follows. In Section II, we provide mathematical preliminaries and some definitions. Our main results are presented in Section III. In Section IV, we present the unicycle case study. Most technical proofs are included in the appendices.

\section{PRELIMINARIES}

A function $\alpha: \mathbb{R}_{>0} \rightarrow \mathbb{R}_{>0}$ is said to be of class $\mathcal{K}(\alpha \in \mathcal{K})$, if it is continuous, strictly increasing and zero at zero; $\alpha \in \mathcal{K}_{\infty}$ if, in addition, it is unbounded. A function $\beta: \mathbb{R}_{\geq 0} \times \mathbb{R}_{\geq 0} \rightarrow$ $\mathbb{R}_{\geq 0}$ is of class $\mathcal{K} \mathcal{L}$ if for all $t>0, \beta(\cdot, t) \in \mathcal{K}$ and for all $s>0, \beta(s, \cdot)$ is decreasing to zero. A function $\gamma: \mathbb{R}_{>0} \rightarrow \mathbb{R}_{>0}$ is said to be of class $\mathcal{N}$ if $\gamma(\cdot)$ is continuous and nondecreasing. We denote by $|\cdot|$ the Euclidean norm of vectors. We denote by $\mathbb{R}$ and $\mathbb{N}$ the sets of the real and natural numbers respectively. For an arbitrary $r \in \mathbb{R}$ we use the notation $\lfloor r\rfloor:=\max _{z \in \mathbb{Z}, z \leq r} z$. Given strictly positive real numbers $L$ and $T$, we use the following notation:

$$
\ell_{L, T}:=\left\lfloor\frac{L}{T}\right\rfloor
$$

For the cascade (1), (2) we use the notation $\xi:=\left[\begin{array}{ll}x^{T} & z^{T}\end{array}\right]^{T}$ to denote the state of the overall system. Often, we regard $z$ in the system (1) as an exogenous input that is not necessarily generated by the subsystem (2). In that case, we refer to the subsystem (1) as the system with input $z$. The solution of the system (1) with input $z$ at time $k$, that starts at initial time instant $k_{\circ}$ from the initial state $x\left(k_{\circ}\right)=x_{\circ}$ and under the action of the input sequence $\omega_{\left[k_{\circ}, k\right)}^{z}:=\left\{z\left(k_{\circ}\right), \ldots z(k-1)\right\}$ is denoted as $\phi_{T}^{x}\left(k, k_{\circ}, x_{\circ}, \omega_{\left[k_{\circ}, k\right)}^{z}\right)$. We also use $\omega^{z}:=\omega_{\left[k_{\circ}, \infty\right)}^{z}$. Note that the solution of the system (3) is the same as the solution for (1) with input $z$ when $z(j) \equiv 0, \forall j \in\left[k_{\circ}, k\right]$ and, hence, for solutions of (3) we use the notation $\phi_{T}^{x}\left(k, k_{\circ}, x_{\circ}, 0\right)$. Similarly we use the notation $\phi_{T}^{\xi}\left(k, k_{\circ}, \xi_{\circ}\right), \phi_{T}^{x}\left(k, k_{\circ}, \xi_{\circ}\right)$ and $\phi_{T}^{z}\left(k, k_{\circ}, z_{\circ}\right)$ to denote solutions of (1) and (2) and of its $x$ and $z$ components respectively.

To state our main results, we need the following assumption and definitions. The motivation to consider properties presented in Definitions 1-4 is to obtain the right type of stability for the parameterized discrete-time system that can be used within the framework ${ }^{1}$ of [34, Th. 1] (to see how these results are used, see also the case study in Section IV).

Assumption 1: There exist $\gamma_{2} \in \mathcal{N}, \gamma_{1}, \gamma_{3} \in \mathcal{K}_{\infty}$ and $T^{*}>0$ such that for all $\xi \in \mathbb{R}^{n}, k \geq 0$ and $T \in\left(0, T^{*}\right)$ we have $\left|f_{T}(k, x, z)\right| \leq \gamma_{1}(|\xi|)$ and $\left|f_{T}(k, x, z)-f_{T}(k, x, 0)\right| \leq T \gamma_{2}(|x|) \gamma_{3}(|z|)$.

Definition 1 [SP-UAS]: The parameterized time-varying system

$$
y(k+1)=F_{T}(k, y(k))
$$

is semiglobally practically uniformly asymptotically stable, i.e., SP-UAS, (respectively, uniformly globally asymptotically stable UGAS) if there exists $\beta \in \mathcal{K} \mathcal{L}$ such that for any pair of strictly positive real numbers $(\Delta, \nu)$ there exists $T^{*}>0$ (respectively, there exists $T^{*}>0$ ) such that for all $k_{\circ} \geq 0, y\left(k_{\circ}\right)=y_{\circ}$ with $\left|y_{\circ}\right| \leq \Delta, T \in\left(0, T^{*}\right)\left(y\left(k_{\circ}\right)=y_{\circ}\right.$ with $\left.y_{\circ} \in \mathbb{R}^{n}, T \in\left(0, T^{*}\right)\right)$ the following holds:

$$
\left|\phi_{T}^{y}\left(k, k_{\circ}, y_{\circ}\right)\right| \leq \max \left\{\beta\left(\left|y_{\circ}\right|,\left(k-k_{\circ}\right) T\right), \nu\right\} \quad \forall k \geq k_{\circ}
$$

(respectively, $\left|\phi_{T}^{y}\left(k, k_{\circ}, y_{\circ}\right)\right| \leq \beta\left(\left|y_{\circ}\right|,\left(k-k_{\circ}\right) T\right)$ ) for all $k \geq$ $\left.k_{\circ}\right)$.

Notice that the convergence of solutions in (6) is not uniform in the sampling period since the solutions are allowed to converge slower as $T$ decreases. However, (6) bounds the overshoots uniformly in $T$.

Definition 2: The parameterized time-varying system (5) is Lyapunov SP-UAS if there exist $\alpha_{1}, \alpha_{2} \in \mathcal{K}_{\infty}, \alpha_{3} \in \mathcal{K}, L \in \mathcal{N}$ and for each pair $(\Delta, \nu)>0$ there exists $T^{*}>0$ and for each

\footnotetext{
${ }^{1}$ Reference [34, Th. 1] is presented for time-invariant systems but it can be stated with minor changes for time-varying systems that we consider in this paper.
} 
$T \in\left(0, T^{*}\right)$ a continuous function $V_{T}: \mathbb{R}_{>0} \times \mathbb{R}^{n} \rightarrow \mathbb{R}_{\geq 0}$ such that for all $|y| \leq \Delta$, all $k \geq 0$ and all $T \in\left(0, T^{*}\right)$ we have that

$$
\begin{aligned}
& \alpha_{1}(|y|) \leq V_{T}(k, y) \leq \alpha_{2}(|y|) \\
& V_{T}\left(k+1, F_{T}(k, y)\right)-V_{T}(k, y) \leq-T\left(\alpha_{3}(|y|)+\nu\right) \\
& \left|V_{T}(k, r)-V_{T}(k, s)\right| \leq L(\max \{|r|,|s|\})|r-s|
\end{aligned}
$$

for all $\max \{|r|,|s|\} \leq \Delta, T \in\left(0, T^{*}\right)$ and $k \geq 0$. The system is Lyapunov UGAS if there exists $T^{*}>0$ such that the aforementioned conditions hold for all $x, r, s \in \mathbb{R}^{n}, k \geq 0$ and with $\nu=0$.

Remark 1: We note that the properties in Definitions 1 and 2 are very related. In particular, it was shown in [34] that the system is SP-UAS (see Definition 1) if and only (7) and (8) hold. However, the converse Lyapunov theorem in [34] does not produce a Lyapunov function satisfying the condition (9) and we believe that constructing such converse Lyapunov functions is an open problem in the literature. In particular, it would be important to provide conditions under which one can construct Lyapunov functions from Definition 2 for (5) with a discontinuous right-hand side. Such converse theorems for nonparameterized discrete-time systems can be found in [14] but we are not aware of similar results for parameterized systems.

Definition 3: The system (5) is uniformly semiglobally bounded (USB), [respectively, uniformly globally bounded (UGB)], if there exist $\kappa \in \mathcal{K}_{\infty}$ and $c$, such that for any $\Delta>0$ there exists $T^{*}>0$ (respectively, there exists $T^{*}>0$ ) such that $k_{\circ} \geq 0, y\left(k_{\circ}\right)=y_{\circ}$ with $\left|y_{\circ}\right| \leq \Delta$ and $T \in\left(0, T^{*}\right)$ $\left(y_{\circ} \in \mathbb{R}^{n}\right.$ and $T \in\left(0, T^{*}\right)$ ) implies

$$
\left|\phi_{T}^{y}\left(k, k_{\circ}, y_{\circ}\right)\right| \leq \kappa\left(\left|y_{\circ}\right|\right)+c
$$

for all $k \geq k_{\circ}$.

The following property is similar to continuity of solutions of differential equations satisfying the local Lipschitz condition if we think of $t:=k T$ as "continuous time." This will be crucial in establishing a trajectory-based proof of our main result.

Definition 4: The solutions of the system (1) with input $z$ are uniformly semiglobally continuous (USC) [uniformly globally continuous (UGC)] if for any $\Delta$ there exists $T^{*}>0$ (there exists $\left.T^{*}>0\right)$ such that for any $\eta \in(0, \Delta), \epsilon>0$ and $L>0$ there exists $\mu>0$ such that for all $T \in\left(0, T^{*}\right)$, all $z(\cdot)$ with $\left|\omega^{z}\right| \leq \mu, k_{\circ} \geq 0$ and all $x\left(k_{\circ}\right)=x_{\circ}$ with $\left|x_{\circ}\right| \leq \eta$ we have that

$$
\begin{array}{r}
\mid \phi_{T}^{x}\left(k, k_{\circ}, x_{\circ}, \omega_{\left[k_{\circ}, k\right)}^{z}\right)- \\
\phi_{T}^{x}\left(k, k_{\circ}, x_{\circ}, 0\right) \mid \leq \epsilon \\
\forall k \in\left[k_{\circ}, k_{\circ}+\ell_{L, T}\right] .
\end{array}
$$

\section{MAIN Results}

In this section, we first present two general results on stability of cascades. In these stability results we make use of all properties presented in Definitions 1-4. Properties in Definitions 1-4 are hard to check in general and, in subsequent Sections III-B and $\mathrm{C}$, we give some easier-to-check sufficient conditions for USC, USB, and Lyapunov SP-UAS.

\section{A. Stability of Parameterized Cascades}

Our main results are presented in this subsection. Two results are presented that deduce stability of the cascade (1) and (2) from stability of two lower dimensional auxiliary systems (3) and (2) and the boundedness of trajectories of the overall system (1) and (2). These results are fundamental in that they are necessary and sufficient for stability of the cascade. Hence, the first condition of [34, Th. 1] can be checked via three separate conditions that are usually easier to verify. Our two main results are similar in spirit but they are derived under slightly different conditions.

Theorem 1: Suppose that the solutions of the system (1) with input $z$ are USC (UGC). Then, the overall system (1) and (2) is SP-UAS (UGAS) if and only if the following conditions hold:

1) system (3) is SP-UAS (UGAS);

2) system (2) is SP-UAS (UGAS);

3 ) system (1) and (2) satisfies the property USB (UGB).

Theorem 2: Suppose that $f_{T}$ of the system (1) satisfies Assumption 1. Then, the overall system (1) and (2) is SP-UAS (UGAS) if the following conditions hold:

1) system (3) is Lyapunov SP-UAS (Lyapunov UGAS);

2) system (2) is SP-UAS (UGAS);

3) system (1) and (2) satisfies the property USB (UGB).

For the sake of clarity, we present the proofs of the main results in Appendix A. We provide only the proofs for the more general case of SP-UAS since the global versions follow by removing the restriction on the size of the domain of attraction and restricting the neighborhood of the origin to the origin itself (i.e., considering $\nu=0$ ).

Remark 2: The proof of Theorem 1 is inspired by the trajectory-based proof in [43, Th. 1] that does not appeal to converse Lyapunov theorems (see Appendix A). On the other hand, Theorem 2 uses Assumption 1 instead of the USC assumption and it is inspired by the proof of [39, Lemma 2] (see Appendix A). We stress that in Theorem 2 we use a different assumption from USC which is typically weaker than the latter. Moreover, we use Lyapunov SP-UAS which is typically stronger than SP-UAS. In particular, in Theorem 2 we may have that the right hand side of (3) is discontinuous, which is in general excluded from Theorem 1 because of the USC condition. Note also that since appropriate converse Lyapunov theorem does not exist for parameterized systems (see Remark 1), we only state sufficiency results in Theorem 2.

Remark 3: While the proof of Theorem 1 does not require the existence of a Lyapunov function, Theorem 2 is important since the existence of a Lyapunov function is often helpful for controller design. Indeed, a Lyapunov function may allow us to improve the transients of the sampled-data system by redesigning a continuous-time controller in an appropriate way and using our results (see Section IV).

Two interesting corollaries for nonparameterized systems follow directly from our results and they generalize some results in [12]. These results are interesting in cases when the exact discrete-time model of the plant can be computed and we 
do not have to appeal to [34, Th. 1]. The appropriate definitions and assumptions for nonparameterized systems

$$
\begin{aligned}
& x(k+1)=f(k, x(k), y(k)) \\
& y(k+1)=g(k, y(k))
\end{aligned}
$$

are obtained easily from global definitions for parameterized systems by setting $T=1$ and they are not repeated for space reasons. Similarly, the auxiliary system is

$$
x(k+1)=f(k, x(k), 0) .
$$

Corollary 1: Suppose that the solutions of the system (12) with input $y$ are UGC. Then, the overall system (12) and (13) is UGAS if and only if

1) system (14) is UGAS;

2) system (13) is UGAS; and

3) system (12), (13) is UGB.

Corollary 2: Suppose that Assumption 1 holds for $f$ in (12). Then, the overall system (12) and (13) is UGAS if

1) system (14) is Lyapunov UGAS;

2) system (13) is UGAS; and

3) system (12), (13) is UGB.

Remark 4: Notice that continuity of $f$ is not required in Corollary 2 whereas we implicitly require continuity in Corollary 1 since this is needed to guarantee UGC (see also Proposition 2).

\section{B. Sufficient Conditions for Lyapunov SP-UAS and for USC}

First, we establish conditions for Lyapunov SP-UAS that are sometimes easier to check that the conditions given in Definition 2. This is illustrated through the case study in next section.

It is often the case that instead of a Lyapunov function that shows that the system is Lyapunov SP-UAS we can find an auxiliary function such that one or more conditions among (7)-(9) does not hold. For instance, for time-varying systems it is often the case that we can find a function $V_{T}$ that satisfies (7) and (9), but instead of (8), it holds that

$$
\frac{\Delta V_{T}}{T}=\frac{V_{T}\left(k+1, F_{T}(k, x)\right)-V_{T}(k, x)}{T} \leq-p_{T}(k) \alpha_{3}(|x|)
$$

where $p_{T}(\cdot)$ is a nonnegative time-varying function that may become zero for some $k \in \mathbb{Z}$. For example, in the case study treated in next section, we have such a situation. It is obvious that under this condition the system is not Lyapunov SP-UAS in general (take, for example, $p_{T}(k) \equiv 0$ ).

In certain cases, it is possible to use results on changes of supply rates and the notion of input-to-state stability, such as [19] and references cited therein. Here, we present another construction that is useful for time-varying systems: we show that if the function $p_{T}(\cdot)$ is persistently exciting in an appropriate sense and there exists a Lyapunov function $V_{T}$ that satisfies (7), (9), and (15), then we can construct a new Lyapunov function $U_{T}$ to show Lyapunov SP-UAS (that is, (7)-(9) hold for $U_{T}$ ).
Our result is similar in spirit to the continuous-time result [26] but our construction is more related to the construction [24, eq. (19)] and [33].

We introduce next a modified definition of persistency of excitation that is tailored for discrete-time parameterized systems to be used within the framework of [34].

Definition 5 (PE): Let $p_{T}: \mathbb{Z}_{\geq 0} \rightarrow \mathbb{R}_{\geq 0}$ be a function produced by sampling a function $\psi: \mathbb{R}_{\geq 0} \rightarrow \mathbb{R}_{\geq 0}$ at rate $T$. The function $p_{T}(\cdot)$ is said to be persistently exciting $(\mathrm{PE})$ if there exist positive numbers $\mu, L$ and $T^{*}$ such that for all $T \in\left(0, T^{*}\right)$ and all $j \geq 0$

$$
T \sum_{k=j}^{j+\ell_{L, T}} p_{T}(k) \geq \mu .
$$

We present the following result only for the case of Lyapunov UGAS since the Lyapunov SP-UAS case follows with minor modifications. The proof is provided in Appendix $\mathrm{C}$.

Proposition 1: Given positive numbers $c_{1}, c_{2}, c_{3}, c_{4}, p_{M}, \hat{T}$, a persistently exciting function $p_{T}(\cdot)$, positive-definite, radially unbounded, and continuous functions $\psi: \mathbb{R}^{n} \rightarrow \mathbb{R}_{\geq 0}$ and $V_{T}: \mathbb{R} \times \mathbb{R}^{n} \rightarrow \mathbb{R}_{\geq 0}$, suppose that the following conditions hold for all $x \in \mathbb{R}^{n}, \bar{T} \in(0, \hat{T})$ and all $k \geq 0$ :

$$
\begin{aligned}
c_{1} \psi(x) \leq V_{T}(k, x) & \leq c_{2} \psi(x) \\
\frac{V_{T}\left(k+1, F_{T}(k, x)\right)-V_{T}(k, x)}{T} & \leq-c_{3} p_{T}(k) \psi(x)+T c_{4} \psi(x) \\
p_{T}(k) & \leq p_{M}
\end{aligned}
$$

Then, there exists $\epsilon^{*}>0$ and for each $\epsilon \in\left(0, \epsilon^{*}\right)$ there exist positive numbers $d_{1}, d_{2}, d_{3}, T^{*}$ such that the function

$$
U_{T}(k, x):=V_{T}(k, x)+\epsilon W_{T}(k, x)
$$

with $W_{T}(k, x):=-T \sum_{i=k}^{\infty} e^{(k-i) T} p_{T}(i) V_{T}(k, x)$, satisfies for all $x \in \mathbb{R}^{n}, T \in\left(0, T^{*}\right)$ and $k \geq 0$

$$
\begin{aligned}
d_{1} \psi(x) \leq U_{T}(k, x) & \leq d_{2} \psi(x) \\
\frac{U_{T}\left(k+1, F_{T}(k, x)\right)-U_{T}(k, x)}{T} & \leq-d_{3} \psi(x) .
\end{aligned}
$$

We note that the aforementioned construction is slightly less general than the one in [26] since all bounds in Proposition 1 use the same function $\psi(x)$. However, this may be relaxed if we use a more general construction (see [26]) of the form $U_{T}(k, x)=$ $\rho_{1}\left(V_{T}\right)+P(k) \rho_{2}\left(V_{T}\right)$ where $\rho_{1}, \rho_{2} \in \mathcal{K}_{\infty}$. We have not pursued this direction for space reasons and since Proposition 1 suffices for our case study. Moreover, it is obvious how one can state a corollary of Proposition 1 that applies to non parameterized discrete-time systems. Furthermore, note that the result on exponential stability is obtained from Proposition 1 by taking $\psi(x)=|x|^{2}$. These results are omitted for space reasons.

We present next, checkable conditions for USC inspired by the literature on numerical methods (cf., [47]).

Proposition 2: Suppose that the system (3) is USB (UGB) and for any pair of strictly positive $\left(\Delta_{1}, \Delta_{2}\right)$ there exist strictly 
positive $K, T^{*}$ such that: For all $k \geq 0, \max \left\{\left|x_{1}\right|,\left|x_{2}\right|\right\} \leq$ $\Delta_{1},\left|z_{1}\right| \leq \Delta_{2}$ and $T \in\left(0, T^{*}\right)$ we have

$$
\begin{aligned}
& \left|f_{T}\left(k, x_{1}, z_{1}\right)-f_{T}\left(k, x_{2}, 0\right)\right| \leq(1+K T)\left|x_{1}-x_{2}\right| \\
& \left|f_{T}\left(k, x_{1}, z_{1}\right)-f_{T}\left(k, x_{1}, 0\right)\right| \leq K T\left|z_{1}-z_{2}\right| .
\end{aligned}
$$

Then, (1) with input $z$ is USC (UGC).

The proof of this proposition is provided in Appendix B.

\section{Sufficient Conditions for USB}

We stress that UGB is in general difficult to check. In [23] we present several sufficient conditions for this property to hold and which are inspired from [3], [39]. For the sake of completeness, we close this section with a result which is representative of the type of conditions given in those references and which we will use later on in the case study. The proof follows along the proofs of [23, Lemmas 1 and 2] and is therefore omitted.

Proposition 3: Consider the system (1) with input $z$. Suppose that there exist $\tilde{\alpha}_{1}, \tilde{\alpha}_{2}, \varphi \in \mathcal{K}_{\infty}, \tilde{\gamma}_{1}, \tilde{\gamma}_{2} \in \mathcal{N}, T^{*}>0, c \geq$ 0 and for each $T \in\left(0, T^{*}\right)$ there exists $V_{T}: \mathbb{R}_{\geq 0} \times \mathbb{R}^{n_{x}} \rightarrow \mathbb{R}_{\geq 0}$ such that for all $x \in \mathbb{R}^{n_{x}}, z \in \mathbb{R}^{n_{z}}, k \geq 0$ and $T \in\left(0, T^{*}\right)$ we have that

$$
\begin{aligned}
\tilde{\alpha}_{1}(|x|) \leq V_{T}(k, x) \leq & \tilde{\alpha}_{2}(|x|)+c \\
V_{T}\left(k+1, f_{T}(k, x, z)\right)- & V_{T}(k, x) \leq \\
& T \tilde{\gamma}_{1}(|z|) \varphi\left(V_{T}(k, x)\right) \\
& +T \tilde{\gamma}_{2}(|z|) \\
\int_{1}^{\infty} \frac{d s}{\varphi(s)}= & \infty
\end{aligned}
$$

If, furthermore, the solutions of (2) satisfy the summability condition

$$
T \sum_{k=k_{\circ}}^{\infty} \mu\left(\left|\phi_{T}^{z}\left(k, k_{\circ}, z_{\circ}\right)\right|\right) \leq \rho\left(\left|z_{\circ}\right|\right)
$$

with some $\rho \in \mathcal{K}_{\infty}$ and $\mu(s):=\tilde{\gamma}_{1}(s)+\tilde{\gamma}_{2}(s) / \varphi(1)$ then, the system (1), (2) is UGB.

This proposition establishes some interesting links with conditions used in the literature in the context of continuous-time systems to prove UGB. Roughly speaking, (26) can be regarded as the combination of a growth rate restriction on $V_{T}(k, \cdot)$ and the interconnection terms (those which depend on $z$ ) present in $f_{T}(k, x, z)$. Such conditions are of standard use in the context of continuous time cascades (see, for instance [41] and [10]).

In the particular case when $\tilde{\gamma}_{1}, \tilde{\gamma}_{2} \in \mathcal{K}_{\infty}$ and $c=0$, one can prove that there exist $\mathcal{K}_{\infty}$ functions $\alpha_{1}, \alpha_{2}$ such that the systems trajectories satisfy

$$
\begin{aligned}
& \alpha_{1}\left(\left|\phi_{T}^{x}\left(k, k_{\circ}, x_{\circ}, \omega_{\left[k_{\circ}, k\right)}^{z}\right)\right|\right) \leq \alpha_{2}\left(\left|x_{\circ}\right|\right) \\
& +T \sum_{i=k_{\circ}}^{k-1} \mu\left(\left|\omega_{\left[k_{\circ}, k\right)}^{z}\right|\right) \quad \forall k \geq k_{\circ} \geq 0
\end{aligned}
$$

which is closely related to the integral input to state stability property [44], [2].

Condition (27) restricts the growth of $\varphi$. In particular, it holds when $\varphi(s)=s$; this situation was considered, for instance, in
[39] with $\tilde{\gamma}_{1}(s) \equiv 0$. Earlier results using similar conditions are found in [27] and as a sufficient condition for forward completeness already in [42].

The summability condition (28), which imposes a minimal convergence rate for the system (2), is tight. However, we are not aware of any proof of necessity. This condition is in general hard to check since it is trajectory-dependent. A similar condition was used in the context of continuous-time cascades in [3] and [38]. Related results for parameterized discrete-time systems are also given in [23] along the lines of the results presented in these references.

\section{Case Study: Tracking Control of the Unicycle}

In this section, we revisit the problem of tracking control of a mobile robot of the unicycle type. This problem has been thoroughly studied in the continuous-time context via many different approaches (see [16] for a survey; for a more recent text with an updated list of references, see [20]). To illustrate the utility of our results we will revisit the cascades approach used in [37] for a three degrees-of-freedom cart. The results may be extended to higher dimension systems, following for instance [20]. According to [13] the context of the problem can be set as follows. We have a mobile robot with two directional wheels and two "fixed" wheels and whose motion is described by

$$
\dot{x}=v \cos \theta ; \quad \dot{y}=v \sin \theta ; \quad \dot{\theta}=\omega
$$

where $x, y$ are the Cartesian coordinates of the center of the axis joining the directional wheels and $\theta$ is the orientation angle of the directional wheels. The robot is required to follow a trajectory generated by an exosystem, i.e., a fictitious "reference robot" with kinematics

$$
\dot{x}_{r}=v_{r}(t) \cos \theta_{r} \quad \dot{y}_{r}=v_{r}(t) \sin \theta_{r} \quad \dot{\theta}_{r}=\omega_{r}(t)
$$

where $v_{r}(t)$ and $\omega_{r}(t)$ are given reference velocities. Then, the tracking errors satisfy the set of equations (see [13, Lemma 1])

$$
\begin{aligned}
& \dot{x}_{e}=\omega y_{e}-v+v_{r}(t) \cos \theta_{e} \\
& \dot{y}_{e}=-\omega x_{e}+v_{r}(t) \sin \theta_{e} \\
& \dot{\theta}_{e}=\omega_{r}(t)-\omega v
\end{aligned}
$$

where $(\cdot)_{e}:=(\cdot)_{r}-(\cdot)$. The system is velocity-controlled, i.e., the control problem reduces to finding control inputs $\omega$ and $v$ (which also correspond to the actual angular and linear velocities of the cart) such that the origin of (32) is UGAS.

There are numerous solutions to this problem in the context of continuous-time (e.g., [11], [30], and [20] for a recent literature review). Here, we will revisit the cascaded approach proposed in [37] whose main feature is that the control laws are linear. Moreover, we use the Euler-discretization of the error dynamics to design a tracking controller

$$
\begin{aligned}
& x_{e}(k+1)=x_{e}(k)+T\left[\omega y_{e}(k)-v+v_{r}(k) \cos \theta_{e}(k)\right] \\
& y_{e}(k+1)=y_{e}(k)+T\left[-\omega x_{e}(k)+v_{r}(k) \sin \theta_{e}(k)\right] \\
& \theta_{e}(k+1)=\theta_{e}(k)+T\left[\omega_{r}(k)-\omega\right] .
\end{aligned}
$$


Thus, our control problem consists in designing $v$ and $\omega$ such that (33) is UGAS. Suppose that controller has the following structure:

$$
\omega:=\omega_{r}(k)+a_{1} \theta_{e}(k) ; \quad v:=v_{r}(k)+a_{2} x_{e}(k)+T \vartheta
$$

where $a_{1}, a_{2}, \omega_{r}(k)$ and $v_{r}(k)$ come from the continuous-time control law proposed in [37] and $\vartheta$ is an extra control input which depends on $k, x_{e}$ and $y_{e}$ and that we design with the aim of improving the system's performance. Note that the closed-loop approximate Euler model (33) and (34) has a cascaded structure

$$
\begin{aligned}
x_{e}(k+1)= & \left.\begin{array}{c}
\left(1-T a_{2}\right) x_{e}(k)+T \omega_{r}(k) y_{e}(k)-T^{2} \vartheta \\
y_{e}(k)-T \omega_{r}(k) x_{e}(k)
\end{array}\right) \\
& +\underbrace{\begin{array}{r}
T\left[a_{1} \theta_{e}(k) y_{e}(k)-v_{r}(k)+v_{r}(k) \cos \theta_{e}(k)\right] \\
T\left[-a_{1} \theta_{e}(k) x_{e}(k)+v_{r}(k) \sin \theta_{e}(k)\right]
\end{array}}_{F_{1 T}(k, x(k))}
\end{aligned}
$$

$\theta_{e}(k+1)=\left(1-T a_{1}\right) \theta_{e}(k)=: F_{2 T}(k, x(k))$

where $z:=\theta$ and $X:=\operatorname{col}\left[x_{e}, y_{e}\right]$. To compact the notation, in the sequel we will use $\omega_{r_{k}}:=\omega_{r}(k)$. Now, we can state the following.

Proposition 4: Consider the system (33) in closed loop with (34). Assume the following

1) There exists $\hat{T}, w_{M}>0$ such that for all $k \geq 0$ and $T \in(0, \hat{T})$

$$
\max \left\{\left|v_{r_{k}}\right|,\left|\omega_{r_{k}}\right|, \frac{\left|\omega_{r_{k}}-\omega_{r_{k-1}}\right|}{T}\right\} \leq w_{M} .
$$

2) The function $\omega_{r}^{2}$ is PE.

Then, there exists $a_{2}>0$ such that for all $K, a_{1}>0$ and $\vartheta(k, X)$ with $|\vartheta(k, X)| \leq K|X|$, the system is UGAS.

The proof of this proposition is given in Appendix D. Using this result, we can summarize the steps in designing the controller.

Step 1) Following a similar approach to that of [37] where it was shown, using results for continuous-time cascaded systems, that the system (32) in closed loop with $v=v_{r}(t)+a_{2} x_{e}$ and $\omega=\omega_{r}(t)+a_{1} \theta_{e}$ is UGAS for appropriately chosen $a_{1}$ and $a_{2}$.

Step 2) Assume that the sampled-data controller has the structure given in (34).

Step 3) Using Proposition 4 (which is proved using Theorem 2 and Proposition 1), we see that under appropriate conditions the closed-loop Euler model with the controller (34) and with zero input, i.e., $\theta_{e}=0$, is SP-UAS for all $\vartheta(k, X)$ satisfying $|\vartheta(k, X)| \leq$ $K|X|$ with $K>0$. Moreover, from the proof of Proposition 4, we obtain a particular Lyapunov function $V_{T}(k, X)$ for the subsystem $\left(x_{e}, y_{e}\right)$.

Step 4) Use the Lyapunov function $V_{T}(k, X)$ as the control Lyapunov function (where $\vartheta$ is the new control input) for the Euler approximate model of the subsystem $\left(x_{e}, y_{e}\right)$ with the controller (34) and design $\vartheta$ so that the decrease of $\Delta V_{T}$ along solutions of the subsystem is enhanced when compared to the situation $\vartheta \equiv 0$. One such choice is

$$
\vartheta(k, X):=\frac{\left(a_{2}^{2}+\omega_{r_{k}}^{2}-\varepsilon a_{2}\right) x_{e}-\left(2 a_{2} \omega_{r_{k}}-\varepsilon \omega_{r_{k}}^{3}\right) y_{e}}{2\left(1-a_{2} T\right)+\varepsilon \omega_{r_{k}}^{2} T}
$$

with $\varepsilon=\alpha_{y}+T$.

Step 5) Using [31, Th. 1] (see also [34, Th. 1]), we conclude that the exact closed-loop discrete-time system is stable for sufficiently small $T$ with $\vartheta$ designed in Step 4).

Remark 5: Although the unicycle system is simple at first sight, the use of Proposition 1 is fundamental to the analysis above since, in contrast to the continuous-time setting, the system is not passive from an external additive input. More precisely, notice that (26) does not hold with a simple quadratic function as is the case for the continuous-time model. Hence, even the property of UGB is not simple to verify in general.

We emphasize that the aforementioned steps can be interpreted as a redesign of the continuous-time controller obtained in [37]. Moreover, the redesign is based on the Euler approximate model of the error dynamics and our rigorous proofs show that this controller would work well for small sampling periods. Furthermore, the simulations that we present next show that the performance is considerably improved as we could expect from the way that $\vartheta$ was designed in Step 4). We have simulated the system above in SIMULINK of MATLAB with $a_{2}=70, a_{1}=$ $10, w_{r}(k)=20 \sin (k T), T=0.01$ and $\alpha_{y}=2-T$. We show the results in Fig. 1. We show only the responses for the states $x_{e}$ and $y_{e}$ as well as $v$ since these are the only variables affected by the additional input $T \vartheta$. We show simulations for the system's response with $\vartheta=0$, and for $\vartheta$ as defined in (38). The best apparent performance is for the latter. It is also clear from the plots, that even though the correction $\vartheta$ is linear in the state and actually $(\vartheta \approx \mathcal{O}(1)|X|)$, this correction is not comparable to "adding gain" to the control input. Notice that in this case the resulting control effort is actually smaller than in the case of the continuous-time based controller (i.e., when $\vartheta=0$ ).

\section{CONCLUSION}

We have established necessary and sufficient conditions for semiglobal practical asymptotic stability of time-varying parameterized cascades. Parameterized systems arise naturally when an approximate discrete-time model of a sampled-data plant is used for controller design. Our results provide the controller designer with a range of tools that can be used for a systematic digital controller design based on approximate discrete-time models. We also proved an auxiliary result on constructing strict Lyapunov functions from nonstrict ones and we believe that this result is of independent interest. The utility of our results was illustrated with a case study, where we have obtained a controller that performs better than the emulated (discretized) continuous-time controller designed in [37] and implemented using a sampler and zero-order-hold. 

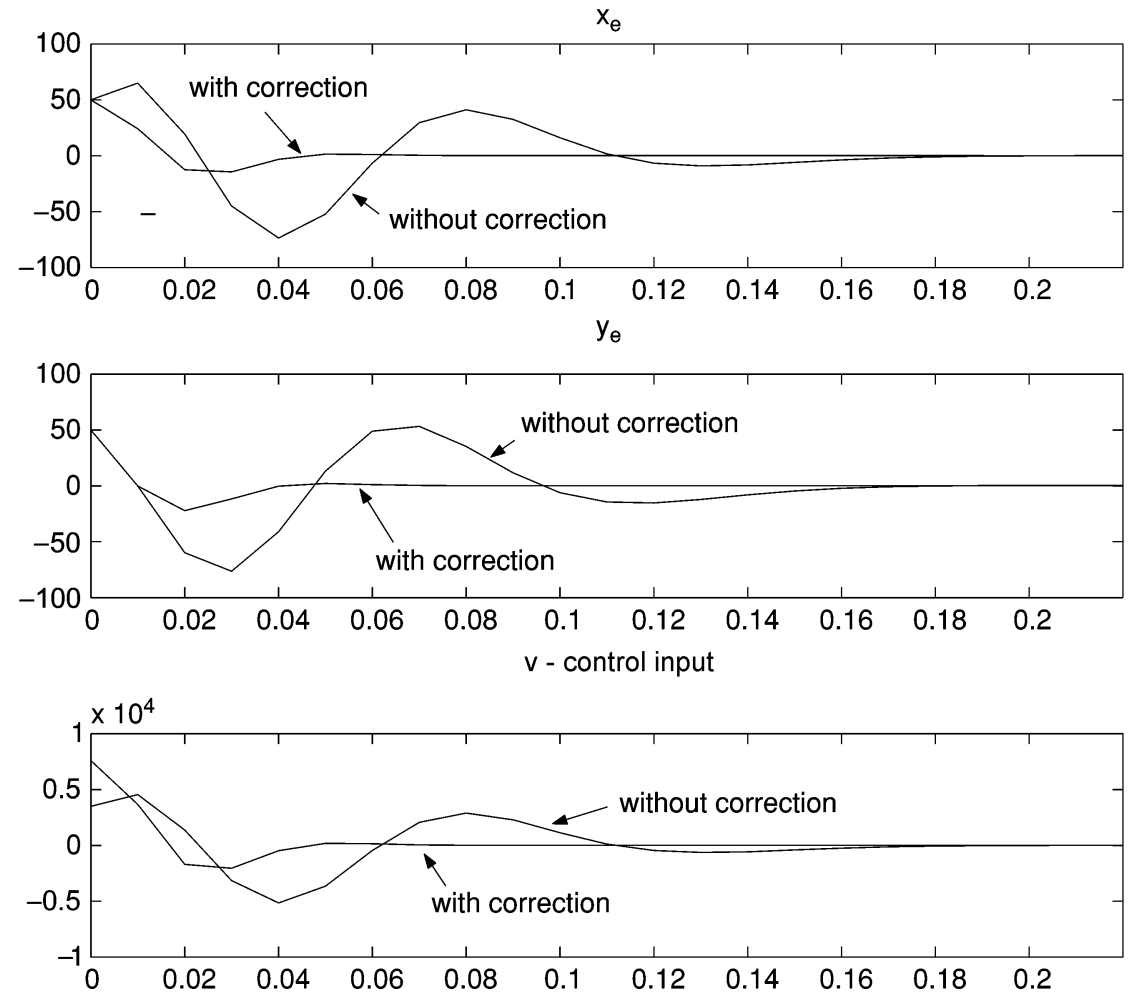

Fig. 1. Tracking errors for $x$ and $y$ and the control input $v$ given by (34), (38).

APPENDIX A

\section{Proofs of THE MAIN THEOREMS}

All results are only proved for semiglobal-practical stability properties since global results follow the same steps with minor changes.

\section{A. Proof of Theorem 1}

Lemma 1: Suppose that the solutions of the system (1) with input $z$ are USC (UGC) and the system (3) is SP-UAS (UGAS), with the function $\beta_{x} \in \mathcal{K} \mathcal{L}$. Then, for any strictly positive $(\Delta, \nu)$ there exists $T^{*}>0$ (there exists $\left.T^{*}\right)$ such that for any $\epsilon>0$ and $\eta \in(0, \Delta]$ (any $\eta>0$ ) there exists $\mu>0$ such that for any input $z(\cdot)$ with $\left|\omega^{z}\right|<\mu, k_{\circ} \geq 0, x\left(k_{\circ}\right)=x_{\circ}$ with $\left|x_{\circ}\right| \leq \Delta$ and $T \in\left(0, T^{*}\right)$ we have that the solutions of the system (1) with input $z$ satisfy the inequality

$\left|\phi_{T}^{x}\left(k, k_{\circ}, x_{\circ}, \omega_{\left[k_{\circ}, k\right)}^{z}\right)\right| \leq \max \left\{\beta_{x}\left(\left|x_{\circ}\right|,\left(k-k_{\circ}\right) T\right), \nu\right\}+\epsilon$ $\forall k \geq k_{\circ}$.

Proof: Note first that for any $(\Delta, \nu)$ there exists $T^{*}>$ 0 such that for any $\eta \in(0, \Delta]$ and any strictly positive real numbers $(\epsilon, L)$ there exists $\mu>0$ such that for all inputs $z(\cdot)$ with $\left|\omega^{z}\right| \leq \mu$, all $k_{\circ} \geq 0, x\left(k_{\circ}\right)=x_{\circ}$ with $\left|x_{\circ}\right| \leq \eta$ and $T \in\left(0, T^{*}\right)$ the following holds:

$$
\begin{aligned}
&\left|\phi_{T}^{x}\left(k, k_{\circ}, x_{\circ}, \omega_{\left[k_{\circ}, k\right)}^{z}\right)\right| \leq\left|\phi_{T}^{x}\left(k, k_{\circ}, x_{\circ}, 0\right)\right| \\
&+ \mid \phi_{T}^{x}\left(k, k_{\circ}, x_{\circ}, \omega_{\left[k_{\circ}, k\right)}^{z}\right) \\
&-\phi_{T}^{x}\left(k, k_{\circ}, x_{\circ}, 0\right) \mid \\
& \leq \max \left\{\beta_{x}\left(\left|x_{\circ}\right|,\left(k-k_{\circ}\right) T\right), \nu\right\}+\epsilon \\
& \forall k \in\left[k_{\circ}, k_{\circ}+\ell_{L, T}\right]
\end{aligned}
$$

Hence, from (11) and the $\mathcal{K} \mathcal{L}$ bound on $\left|\phi_{T}^{x}\left(k, k_{\circ}, x_{\circ}, 0\right)\right|$, it follows that (39) holds for all $k \in\left[k_{\circ}, k_{\circ}+\ell_{L, T}\right]$.

Next, we show that (11) holds for all $k \geq k_{0}$. Let $\beta_{x} \in$ $\mathcal{K} \mathcal{L}$ come from SP-UAS stability of (3). Let $(\Delta, \nu)$ be given. Without loss of generality, assume that $\Delta>\nu$ and

$$
\beta_{x}(s, 0) \geq s \quad \forall s \geq 0 .
$$

Let $\tilde{\nu} \in(0,1)$ be such that

$$
\beta_{x}(\tilde{\nu}, 0)+\tilde{\nu} / 2 \leq \nu
$$

Note that (41) implies that $\tilde{\nu}<\nu$. Let $(\Delta, \tilde{\nu} / 2)$ generate $T^{*}>0$ via (40) and assume without loss of generality that $T^{*}<1$. Let $\epsilon>0$ and $\eta \in(0, \Delta]$ be given. Let $\delta_{1}>0$ be such that

$$
\beta_{x}\left(\delta_{1}, 0\right)+\delta_{1} / 2 \leq \epsilon .
$$

Let $L>1$ be such that

$$
\beta_{x}\left(\max \left\{\eta, \delta_{1}, 1\right\}, L-1\right)<\frac{\delta_{1}}{2} .
$$

Let $\epsilon_{1}:=\min \left\{\tilde{\nu} / 2, \delta_{1} / 2\right\}$ and let $\epsilon_{1}$ and $L$ generate $\mu>0$ via (40). In the rest of the proof, we consider arbitrary fixed $k_{\circ} \geq 0, x\left(k_{\circ}\right)=x_{\circ}$ with $\left|x_{\circ}\right| \leq \eta,\left|\omega^{z}\right| \leq \mu$ and $T \in\left(0, T^{*}\right)$.

In order to simplify the notation, for $i \in \mathbb{Z}_{\geq 1}$ we introduce

$$
k_{i}:=k_{\circ}+i \ell_{L, T} \quad x_{i}:=\phi_{T}^{x}\left(k_{i}, k_{\circ}, x_{\circ}, \omega_{\left[k_{\circ}, k_{i}\right)}^{z}\right) .
$$

Time Interval $\left[k_{\circ}, k_{1}\right]$ : From (40)-(42), we can write

$$
\begin{aligned}
& \left|\phi_{T}^{x}\left(k, k_{\circ}, x_{\circ}, \omega_{\left[k_{\circ}, k\right)}^{z}\right)\right| \\
& \quad \leq \max \left\{\beta_{x}\left(\left|x_{\circ}\right|,\left(k-k_{\circ}\right) T\right), \tilde{\nu} / 2\right\}+\epsilon_{1} \\
& \quad \leq \max \left\{\beta_{x}\left(\left|x_{\circ}\right|,\left(k-k_{\circ}\right) T\right), \nu\right\}+\epsilon
\end{aligned}
$$


for all $k \in\left[k_{\circ}, k_{1}\right]$. Moreover, note that

$$
T<1 \Longrightarrow L-1 \leq T \ell_{L, T}
$$

Using, (44), (46), and our choice of $\epsilon_{1}$, we can write

$$
\begin{aligned}
\left|x_{1}\right| & =\left|\phi_{T}^{x}\left(k_{1}, k_{\circ}, x_{\circ}, \omega_{\left[k_{\circ}, k_{1}\right)}^{z}\right)\right| \\
& \leq \max \left\{\beta_{x}\left(\left|x_{\circ}\right|, T \ell_{L, T}\right), \tilde{\nu} / 2\right\}+\epsilon_{1} \\
& \leq \max \left\{\beta_{x}(\eta, L-1), \tilde{\nu} / 2\right\}+\epsilon_{1} \\
& \leq \max \left\{\delta_{1} / 2, \tilde{\nu} / 2\right\}+\min \left\{\delta_{1} / 2, \tilde{\nu} / 2\right\} \\
& \leq \max \left\{\delta_{1}, \tilde{\nu}\right\} .
\end{aligned}
$$

Time Interval $\left[k_{1}, k_{2}\right]:$ Since $\left|x_{1}\right| \leq \max \left\{\delta_{1}, \tilde{\nu}\right\}$, we consider two cases.

Case 1) $\left|x_{1}\right| \leq \tilde{\nu}$. In this case, using (40)-(42), we can write

$$
\begin{aligned}
& \left|\phi_{T}^{x}\left(k, k_{\circ}, x_{\circ}, \omega_{\left[k_{\circ}, k\right)}^{z}\right)\right| \\
& \quad=\left|\phi_{T}^{x}\left(k, k_{1}, x_{1}, \omega_{\left[k_{1}, k\right)}^{z}\right)\right| \\
& \quad \leq \max \left\{\beta_{x}\left(\left|x_{1}\right|, 0\right), \tilde{\nu} / 2\right\}+\epsilon_{1} \\
& \quad \leq \max \left\{\beta_{x}(\tilde{\nu}, 0), \tilde{\nu} / 2\right\}+\min \left\{\tilde{\nu} / 2, \delta_{1} / 2\right\} \\
& \quad \leq \beta_{x}(\tilde{\nu}, 0)+\tilde{\nu} / 2 \leq \nu
\end{aligned}
$$

for all $k \in\left[k_{1}, k_{2}\right]$. Moreover, using (47), the fact that $\tilde{\nu}<1$, (44), (46), and our choice of $\epsilon_{1}$, we can write

$$
\begin{aligned}
& \left|\phi_{T}^{x}\left(k_{2}, k_{\circ}, x_{\circ}, \omega_{\left[k_{\circ}, k_{2}\right)}^{z}\right)\right| \\
& \quad \leq \max \left\{\beta_{x}\left(\left|x_{1}\right|, T \ell_{L, T}\right), \tilde{\nu} / 2\right\}+\epsilon_{1} \\
& \quad \leq \max \left\{\beta_{x}(\tilde{\nu}, L-1), \tilde{\nu} / 2\right\}+\epsilon_{1} \\
& \quad \leq \max \left\{\beta_{x}(1, L-1), \tilde{\nu} / 2\right\}+\epsilon_{1} \\
& \quad \leq \max \left\{\delta_{1} / 2, \tilde{\nu} / 2\right\}+\min \left\{\delta_{1} / 2, \tilde{\nu} / 2\right\} \leq \max \left\{\delta_{1}, \tilde{\nu}\right\} .
\end{aligned}
$$

Case 2) $\left|x_{1}\right| \leq \delta_{1}$. Using (40) and (43), we can write

$$
\begin{aligned}
& \left|\phi_{T}^{x}\left(k, k_{\circ}, x_{\circ}, \omega_{\left[k_{\circ}, k\right)}^{z}\right)\right| \\
& \quad=\left|\phi_{T}^{x}\left(k, k_{1}, x_{1}, \omega_{\left[k_{1}, k\right)}^{z}\right)\right| \\
& \quad \leq \max \left\{\beta_{x}\left(\delta_{1}, 0\right), \tilde{\nu} / 2\right\}+\min \left\{\tilde{\nu} / 2, \delta_{1} / 2\right\} \\
& \quad \leq \max \left\{\beta_{x}\left(\delta_{1}, 0\right)+\delta_{1} / 2, \tilde{\nu}\right\} \leq \max \{\epsilon, \tilde{\nu}\}
\end{aligned}
$$

for all $k \in\left[k_{1}, k_{2}\right]$. Also, in a similar manner as before, it follows that

$$
\begin{aligned}
& \left|\phi_{T}^{x}\left(k_{2}, k_{\circ}, x_{\circ}, \omega_{\left[k_{\circ}, k_{2}\right)}^{z}\right)\right| \\
& \quad=\left|\phi_{T}^{x}\left(k_{2}, k_{1}, x_{1}, \omega_{\left[k_{1}, k_{2}\right)}^{z}\right)\right| \\
& \quad \leq \max \left\{\beta_{x}\left(\delta_{1}, T \ell_{L, T}\right), \tilde{\nu} / 2\right\}+\epsilon_{1} \\
& \quad \leq \max \left\{\beta_{x}\left(\delta_{1}, L-1\right), \tilde{\nu} / 2\right\}+\epsilon_{1} \\
& \quad \leq \max \left\{\delta_{1} / 2, \tilde{\nu}\right\}+\min \left\{\tilde{\nu} / 2, \delta_{1} / 2\right\} \\
& \quad \leq \max \left\{\delta_{1}, \tilde{\nu}\right\} .
\end{aligned}
$$

Time Intervals $\left[k_{i}, k_{i+1}\right], i \geq 1$ : Using similar calculations it can be shown by induction that for all integers $i \geq 1$

$$
\left|\phi_{T}^{x}\left(k, k_{\circ}, x_{\circ}, \omega_{\left[k_{\circ}, k\right)}^{z}\right)\right| \leq \max \{\epsilon, \nu\} \quad \forall k \in\left[k_{i}, k_{i+1}\right]
$$

$$
\left|\phi_{T}^{x}\left(k_{i}, k_{\circ}, x_{\circ}, \omega_{\left[k_{\circ}, k_{i}\right)}^{z}\right)\right| \leq \max \left\{\delta_{1}, \tilde{\nu}\right\} .
$$

The proof follows from (45) and (55) by noting that

$$
\begin{aligned}
& \left|\phi_{T}^{x}\left(k, k_{\circ}, x_{\circ}, \omega_{\left[k_{\circ}, k\right)}^{z}\right)\right| \\
& \quad \leq \max \{\epsilon, \nu\} \leq \epsilon+\nu \\
& \quad \leq \max \left\{\beta_{x}\left(\left|x_{\circ}\right|,\left(k-k_{\circ}\right) T\right), \nu\right\}+\epsilon \quad \forall k \geq k_{1} .
\end{aligned}
$$

Lemma 2: Suppose that all conditions of Lemma 1 hold and $\beta_{x} \in \mathcal{K} \mathcal{L}$ comes from SP-UAS (UGAS) of (3). Then, there exist $c_{x}, c_{u}>0, \gamma \in \mathcal{K}$ and for any $\nu>0$ there exists $T^{*}>0$ such that for all $k_{\circ} \geq 0, x\left(k_{\circ}\right)=x_{\circ}$ with $\left|x_{\circ}\right| \leq c_{x},\left|\omega^{z}\right|<c_{u}$ and $T \in\left(0, T^{*}\right)$, the following holds:

$$
\begin{array}{r}
\left|\phi_{T}^{x}\left(k, k_{\circ}, x_{\circ}, \omega_{\left[k_{\circ}, k\right)}^{z}\right)\right| \leq \max \left\{\beta_{x}\left(\left|x_{\circ}\right|,\left(k-k_{\circ}\right) T\right), \nu\right\} \\
+\gamma\left(\left|\omega_{\left[k_{\circ}, k\right)}^{z}\right|\right)
\end{array}
$$

for all $k \geq k_{\circ} \geq 0$.

Proof: The proof of this fact follows closely the proof of [15, Lemma 3.3]. Since also all conditions of Lemma 1 hold, the conclusion of Lemma 1 holds. Let $\left(c_{x}, \nu\right)$ generate $T^{*}>0$ via Lemma 1. Let $\eta=c_{x}$ and for a fixed $\epsilon>0$, let $\bar{\mu}(\epsilon)$ be the supremum over all applicable $\mu$ 's. Then, $\left|\omega_{\left[k_{\circ}, k\right)}^{z}\right|<\bar{\mu}(\epsilon)$ implies that (39) holds for all $k \geq k_{\circ} \geq 0$ and if $\mu_{1}>\bar{\mu}(\epsilon)$ then there exists $\left|\bar{\omega}_{\left[k_{\circ}, k\right)}^{z}\right|<\mu_{1}$ and $\left|\phi_{T}^{x}\left(k, k_{\circ}, x_{\circ}, \bar{\omega}_{\left[k_{\circ}, k\right)}^{z}\right)\right|>$ $\max \left\{\beta_{x}\left(\left|x_{\circ}\right|,\left(k-k_{\circ}\right) T\right), \nu\right\}+\epsilon \cdot \bar{\mu}(\epsilon)$ is positive and nondecreasing but it is not necessarily continuous. Chose $\zeta \in \mathcal{K}$ such that $\zeta(r) \leq k \bar{\mu}(r)$, with $k \in(0,1)$. Let $\gamma(r)=\zeta^{-1}(r)$ and note that $\gamma \in \mathcal{K}$. Let $c_{u}:=\lim _{r \rightarrow \infty} \zeta(r)$.

Let $\left|x_{0}\right| \leq c_{x}, T \in\left(0, T^{*}\right)$. Given $\omega_{\left[k_{\circ}, k\right)}^{z}$ with $\left|\omega_{\left[k_{0}, k\right)}^{z}\right|<c_{u}$ let $\epsilon=\gamma\left(\left|\omega_{\left[k_{0}, k\right)}^{z}\right|\right)$. Then, we have that (58) holds for all $k \geq$ $k_{\circ} \geq 0$.

Remark 6: The following fact was proved in [35]: for any $\beta \in \mathcal{K} \mathcal{L}$ and any $c>0$ there exists $\tilde{\beta} \in \mathcal{K} \mathcal{L}$ such that

$$
\beta(s, t) \leq \tilde{\beta}(s, t+c) \quad \forall s, \quad t \in[0, \infty) .
$$

This can be further strengthened in the following manner. It was shown in [44] that given any $\beta \in \mathcal{K} \mathcal{L}$, there exist $\sigma \in \mathcal{K}_{\infty}, \kappa \in$ $\mathcal{K}$ such that $\beta(r, t) \leq \sigma\left(\kappa(r) e^{-t}\right) \forall r, t \geq 0$. Consequently, given any $\beta \in \mathcal{K} \mathcal{L}$ and a nondecreasing function $L(\cdot)$, there exists $\tilde{\beta} \in \mathcal{K} \mathcal{L}$ such that

$$
\beta(s, t) \leq \tilde{\beta}(s, t+L(s)) \quad \forall t \in[0, L(s)]
$$

To show this, let $\beta$ generate $\sigma, \kappa \in \mathcal{K}_{\infty}$ as above. Then $\tilde{\beta}(s, t):=\sigma\left(\kappa(s) e^{L(s)} e^{-t}\right)$ proves the claim.

Lemma 3: Let $\xi=\left(\begin{array}{ll}x^{T} & z^{T}\end{array}\right)^{T}$. Suppose that there exist $\beta_{1}, \beta_{2}, \beta_{3} \in \mathcal{K} \mathcal{L}, \gamma \in \mathcal{K}$ and $c_{0}>0$ such that for all $\nu_{1}>0$, there exists $T^{*}>0$ such that for all 
$\max \left\{\left|u\left(k_{\circ}\right)\right|,\left|\xi\left(k_{\circ}\right)\right|\right\} \leq c_{0}, T \in\left(0, T^{*}\right)$ and $k \geq k_{\circ} \geq 0$ we have

$$
\begin{aligned}
|x(k)| \leq & \max \left\{\beta_{1}\left(\left|x\left(k_{\circ}\right)\right|,\left(k-k_{\circ}\right) T\right), \nu_{1}\right\} \\
& +\gamma\left(\sup _{t \in\left[k_{\circ}, k\right]}|u(t)|\right) \\
|u(k)| \leq & \max \left\{\beta_{2}\left(\left|\xi\left(k_{\circ}\right)\right|,\left(k-k_{\circ}\right) T\right), \nu_{1}\right\} \\
|z(k)| \leq & \max \left\{\beta_{3}\left(\left|z\left(k_{\circ}\right)\right|,\left(k-k_{\circ}\right) T\right), \nu_{1}\right\}
\end{aligned}
$$

where $\xi:=\left(x^{T} z^{T}\right)^{T}$. Then, there exist $\beta \in \mathcal{K} \mathcal{L}$ and $c_{1}>0$ such that for any $\nu>0$ there exists $T_{1}^{*}>0$ such that for all $\xi\left(k_{\circ}\right)=\xi_{\circ}$ with $\left|\xi\left(k_{\circ}\right)\right| \leq c_{1}, T \in\left(0, T^{*}\right)$ and $k \geq k_{\circ} \geq 0$ we have

$$
|\xi(k)| \leq \max \left\{\beta\left(\left|\xi_{\circ}\right|,\left(k-k_{\circ}\right) T\right), \nu\right\} .
$$

In particular, we can take

$$
\begin{aligned}
\beta(s, t)=4 \tilde{\beta}_{1}\left(2 \tilde{\beta}_{1}(s, t / 2)\right. & \left.+2 \gamma\left(\tilde{\beta}_{2}(s, 0)\right), t / 2\right) \\
& +4 \gamma\left(\tilde{\beta}_{2}(s, t / 2)\right)+2 \tilde{\beta}_{3}(s, t)
\end{aligned}
$$

where all $\tilde{\beta}_{i}$ are generated via $\beta_{i}$ and an arbitrary fixed $c>0$ using Remark 6. Moreover, if all the inequalities hold globally, then the conclusion holds globally and we can take

$$
\begin{aligned}
\beta(s, t)=\tilde{\beta}_{1}\left(\tilde{\beta}_{1}(s, t / 2)+\right. & \left.\gamma\left(\tilde{\beta}_{2}(s, 0)\right), t / 2\right) \\
& +\gamma\left(\tilde{\beta}_{2}(s, t / 2)\right)+\tilde{\beta}_{3}(s, t) .
\end{aligned}
$$

The proof of Lemma 3 is omitted since it is published in [32].

Proof of Theorem 1: Let $\beta_{x} \in \mathcal{K} \mathcal{L}$ come from item 1) of Theorem 1. Let $\beta_{z} \in \mathcal{K} \mathcal{L}$ come from item 2) of Theorem 1. Let $\kappa \in \mathcal{K}_{\infty}$ and $c>0$ come from item 3) of Theorem 1. Let $\gamma \in \mathcal{K}$ and $c_{x}, c_{u}$ come from Lemma 2. Let $c_{z}>0$ be such that $\beta_{z}\left(c_{z}, 0\right) \leq c_{u}$ and $c_{\xi}:=\min \left\{c_{z}, c_{x}\right\}$. Let $\bar{\beta} \in \mathcal{K} \mathcal{L}$ and $c_{1}>0$ be generated using $\beta_{1}=\beta_{x}, \beta_{2}=\beta_{3}=\beta_{z}, \gamma$ and $c_{0}=c_{\xi}$ via Lemma 3. Let $\kappa_{1}(s):=\left(\left(c / \kappa\left(c_{1}\right)\right)+1\right) \kappa(s)$. Let $L_{i}: \mathbb{R}_{\geq 0} \rightarrow \mathbb{R}_{\geq 0}, i=1,2$ be continuous nondecreasing functions with $L_{i}(0)=1$ such that for all $\Delta>0$ we have

$$
\begin{aligned}
\beta_{z}\left(\Delta, L_{1}(\Delta)-1\right) & \leq c_{1} \\
\beta_{x}\left(\kappa_{1}(\Delta), L_{2} \circ \kappa_{1}(\Delta)-1\right) & \leq c_{1} / 2 .
\end{aligned}
$$

Let $L(s):=L_{1}(s)+L_{2} \circ \kappa_{1}(s)$. Let $\bar{\beta}$ and $L(s)$ generate $\tilde{\beta}$ via Remark 6. Finally, we define

$\beta(s, t):=\max \left\{\tilde{\beta}\left(\kappa_{1}(s), t\right), \bar{\beta}(s, t), \kappa_{1}(s) e^{L_{1}(s)+L_{2} \circ \kappa_{1}(s)} e^{-t}\right\}$.

Let $(\Delta, \nu)$ be given. Let $\nu_{1}:=\min \left\{c_{1} / 2, \nu\right\}$. Let $\left(\kappa_{1}(\Delta), \nu_{1}\right)$ generate $T_{1}^{*}>0$ via Lemma 1 ; let $\left(\Delta, \nu_{1}\right)$ generate $T_{2}^{*}>0$ via item 2) of Theorem 1; let $\nu_{1}$ generate $T_{3}^{*}>0$ via Lemma 2; let $\nu_{1}>0$ generate $T_{4}^{*}>0$ via Lemma 3. Let $T^{*}:=$ $\min \left\{T_{1}^{*}, T_{2}^{*}, T_{3}^{*}, T_{4}^{*}, 1\right\}$ and $T \in\left(0, T^{*}\right)$. Let $k_{\circ} \geq 0, \xi\left(k_{\circ}\right)=$ $\xi_{\circ}$ with $\left|\xi_{\circ}\right| \leq \Delta$. We consider two cases.

Case 1) If $\left|\xi_{0}\right| \leq c_{1}$, then by a direct application of Lemmas 2 and 3 we have that

$$
\begin{aligned}
\left|\phi_{T}^{\xi}\left(k, k_{\circ}, \xi_{\circ}\right)\right| & \leq \max \left\{\bar{\beta}\left(\left|\xi_{\circ}\right|,\left(k-k_{\circ}\right) T\right), \nu_{1}\right\} \\
& \leq \max \left\{\bar{\beta}\left(\left|\xi_{\circ}\right|,\left(k-k_{\circ}\right) T\right), \nu\right\}
\end{aligned}
$$

for all $k \geq k_{\circ} \geq 0$.
Case 2) If $\left|\xi_{\circ}\right| \in\left[c_{1}, \Delta\right]$, then since $\left|\xi_{\circ}\right| \geq c_{1}$, we can write using item 3) of Theorem 1

$$
\begin{aligned}
\left|\phi_{T}^{\xi}\left(k, k_{\circ}, \xi_{\circ}\right)\right| \leq & \kappa\left(\left|\xi_{\circ}\right|\right)+c \\
& \leq \kappa\left(\left|\xi_{\circ}\right|\right)+\frac{c}{\kappa\left(c_{1}\right)} \kappa\left(\left|\xi_{\circ}\right|\right)=\kappa_{1}\left(\left|\xi_{\circ}\right|\right)
\end{aligned}
$$

for all $k \geq k_{\circ} \geq 0$. Hence, we can write that for all $k \in\left[k_{\circ}, k_{\circ}+\ell_{L_{1}, T}+\ell_{L_{2}, T}\right]$ :

$$
\begin{aligned}
& \left|\phi_{T}^{\xi}\left(k, k_{\circ}, \xi_{\circ}\right)\right| \\
& \quad \leq \kappa_{1}\left(\left|\xi_{\circ}\right|\right) \leq \kappa_{1}\left(\left|\xi_{\circ}\right|\right) e^{L_{1}\left(\left|\xi_{\circ}\right|\right)+L_{2} \circ \kappa_{1}\left(\left|\xi_{\circ}\right|\right)} e^{-\left(k-k_{\circ}\right) T} .
\end{aligned}
$$

Using definitions of $\ell_{L_{i}, T}$ and the fact that $T<T^{*}<1$, we obtain that

$$
L_{i}-1 \leq T \ell_{L_{i}, T} \leq L_{i}, \quad i=1,2 .
$$

Moreover, the definitions of $L_{1}, \nu$ we get that for all $k \geq k_{\circ}+$ $\ell_{L_{1}, T}$

$$
\begin{aligned}
\left|\phi_{T}^{z}\left(k, k_{\circ}, z_{\circ}\right)\right| & \leq \max \left\{\beta_{z}\left(\Delta, T \ell_{L_{1}, T}\right), \nu\right\} \\
& \leq \max \left\{\beta_{z}\left(\Delta, L_{1}(\Delta)-1\right), \nu\right\} \leq c_{1} .
\end{aligned}
$$

Furthermore, by letting $k_{1}:=k_{\circ}+\ell_{L_{1}, T}, k_{2}:=$ $k_{\circ}+\ell_{L_{1}, T}+\ell_{L_{2}, T}, x_{1}:=\phi_{T}^{x}\left(k_{1}, k_{\circ}, x_{\circ}, \omega_{\left[k_{\circ}, k_{1}\right)}^{z}\right)$ and $x_{2}=\phi_{T}^{x}\left(k_{2}, k_{\circ}, x_{\circ}, \omega_{\left[k_{\circ}, k_{2}\right)}^{z}\right)$ we obtain that

$$
\begin{aligned}
& \left|\phi_{T}^{x}\left(k_{2}, k_{\circ}, x_{\circ}, \omega_{\left[k_{\circ}, k_{2}\right)}^{z}\right)\right| \\
& \quad=\left|\phi_{T}^{x}\left(k_{2}, k_{1}, x_{1}, \omega_{\left[k_{1}, k_{2}\right)}^{z}\right)\right| \\
& \quad \leq \max \left\{\beta_{x}\left(\left|x_{1}\right|,\left(k_{2}-k_{1}\right) T\right), \nu_{1}\right\}+\epsilon \\
& \quad \leq \max \left\{\beta_{x}\left(\kappa_{1}(\Delta), T \ell_{L_{2}, T}\right), \nu_{1}\right\}+c_{1} / 2 \\
& \quad \leq \max \left\{\beta_{x}\left(\kappa_{1}(\Delta), L_{2} \circ \kappa_{1}(\Delta)-1\right), \nu_{1}\right\}+c_{1} / 2 \\
& \quad \leq \max \left\{c_{1} / 2, c_{1} / 2\right\}+c_{1} / 2=c_{1} .
\end{aligned}
$$

Hence, using (68), (69), Lemmas 2 and 3, and the construction of $\tilde{\beta}$, we have that for all $k \geq k_{2}$ :

$$
\begin{aligned}
& \left|\phi_{T}^{\xi}\left(k, k_{\circ}, \xi_{\circ}\right)\right| \\
& \quad=\left|\phi_{T}^{\xi}\left(k, k_{2}, \xi_{2}\right)\right| \\
& \quad \leq \max \left\{\bar{\beta}\left(\left|\xi_{2}\right|,\left(k-k_{2}\right) T\right), \nu_{1}\right\} \\
& \quad \leq \max \left\{\bar{\beta}\left(\kappa_{1}\left(\left|\xi_{\circ}\right|\right),\left(k-k_{\circ}-\ell_{L_{1}, T}-\ell_{L_{2}, T}\right) T\right), \nu_{1}\right\} \\
& \quad \leq \max \left\{\bar{\beta}\left(\kappa_{1}\left(\left|\xi_{\circ}\right|\right),\left(k-k_{\circ}\right) T-L_{1}-L_{2}\right), \nu_{1}\right\} \\
& \quad \leq \max \left\{\tilde{\beta}\left(\kappa_{1}\left(\left|\xi_{\circ}\right|\right),\left(k-k_{\circ}\right) T\right), \nu\right\}
\end{aligned}
$$

which proves the result with the defined $\beta$.

\section{B. Proof of Theorem 2}

Lemma 4: Suppose that all conditions of Theorem 2 are satisfied. Let $V_{T}$ come from item 1) of Theorem 2 and define

$$
y(k, \xi):=V_{T}\left(k+1, f_{T}(k, x, z)\right)-V_{T}\left(k+1, f_{T}(k, x, 0)\right) .
$$


Then, there exists $\beta_{y} \in \mathcal{K} \mathcal{L}$ such that for all $(\Delta, \nu)$ there exists $T^{*}>0$ such that for all $k_{\circ} \geq 0, \xi\left(k_{\circ}\right)=\xi_{\circ}$ with $\left|\xi_{\circ}\right| \leq \Delta$ and $T \in\left(0, T^{*}\right)$ we have that

$$
\left|y\left(k, \phi_{T}^{\xi}\left(k, k_{\circ}, \xi_{\circ}\right)\right)\right| \leq T \cdot \max \left\{\beta_{y}\left(\left|\xi_{\circ}\right|,\left(k-k_{\circ}\right) T\right), \nu\right\} .
$$

for all $k \geq k_{\circ}$.

The proof of Lemma 4 is omitted since it is published in [32].

Proof of Theorem 2: We only show that there exists $\beta_{x} \in$ $\mathcal{K} \mathcal{L}$ and $\gamma \in \mathcal{K}$ such that for any $(\Delta, \nu)$ there exists $T^{*}>0$ such that for all $k_{\circ} \geq 0, \xi\left(k_{\circ}\right)=\xi_{\circ}$ with $\left|\xi_{\circ}\right| \leq \Delta$ and $T \in\left(0, T^{*}\right)$ we have that

$$
\begin{aligned}
\left|\phi_{T}^{x}\left(k, k_{\circ}, x_{\circ}, \omega^{z}\right)\right| \leq \max \left\{\beta _ { x } \left(\left|x_{\circ}\right|,(\right.\right. & \left.\left.\left.-k_{\circ}\right) T\right), \nu\right\} \\
& +\gamma\left(\left|\omega_{\left[k_{\circ}, k\right)}^{z}\right|\right)
\end{aligned}
$$

for all $k \geq k_{\circ}$, where $u(k):=\left(y\left(k, \phi_{T}^{\xi}\left(k, k_{\circ}, \xi_{\circ}\right)\right)\right) /(T)$ and $\omega^{z}$ is a sequence of $u(k)$ 's on the interval $\left[k_{0}, k\right)$. Then, the conclusion of the Theorem follows directly from Lemma 3 , item 2 of Theorem 2 and Lemma 4.

Let $\alpha_{1}, \alpha_{2}, \alpha_{3}$ come from item 1) of Theorem 2. Define $\alpha(\cdot):=(1 / 2) \alpha_{3} \circ \alpha_{2}^{-1}(\cdot)$ and consider the differential equation

$$
\dot{\zeta}=-\alpha(\eta) \quad \zeta(0)=\zeta_{\circ} .
$$

Without loss of generality we can assume that $\alpha$ is locally a Lipschitz function (see [15, p.139]) and from [45, Lemma 2.5], we have that there exists $\beta \in \mathcal{K} \mathcal{L}$ such that for all $0 \leq \zeta_{0}$ the solution of the differential equation exists, it is unique and satisfies:

$$
\zeta(t)=\beta\left(\zeta_{\circ}, t-t_{\circ}\right) \quad \forall t \geq t_{\circ} \geq 0 .
$$

Define $\tilde{\alpha}(s):=\alpha^{-1}(s)+s$ and:

$$
\begin{aligned}
\beta_{y}(s, t) & :=\alpha_{1}^{-1}\left(\beta\left(\alpha_{2}(s), t\right)\right) \\
\gamma(s) & :=\alpha_{1}^{-1} \circ \tilde{\alpha}(2 s) .
\end{aligned}
$$

Let $(\Delta, \nu)$ be given. Let $\Delta_{1}:=\kappa(\Delta)+c$, where $\kappa, c$ come from the item 3) of Theorem 2. Let $\nu_{1}:=(1 / 2) \tilde{\alpha}^{-1} \circ \alpha_{1}(\nu)$. Let $\left(\Delta_{1}, \nu_{1}\right)$ generate $T^{*} \in(0,1)$ be so that the items 1$)$ and 3$)$ of Theorem 2 hold. Consider arbitrary fixed $k_{\circ} \geq 0, \xi\left(k_{\circ}\right)=\xi_{\circ}$ with $\left|\xi_{\circ}\right| \leq \Delta$ and $T \in\left(0, T^{*}\right)$. Note that the item 3 of Theorem 2 guarantees that the solutions of (1), (2) exist for all $k \geq k_{\circ} \geq 0$ and satisfy $\left|\phi_{T}^{\xi}\left(k, k_{\circ}, \xi_{\circ}\right)\right| \leq \Delta_{1}$. We can write

$$
\begin{aligned}
& V_{T}\left(k+1, f_{T}(k, x, z)\right)-V_{T}(k, x) \\
&= V_{T}\left(k+1, f_{T}(k, x, 0)\right)-V_{T}(k, x) \\
&+V_{T}\left(k+1, f_{T}(k, x, z)\right)-V_{T}\left(k+1, f_{T}(k, x, 0)\right) \\
&= V_{T}\left(k+1, f_{T}(k, x, 0)\right)-V_{T}(k, x)+T u(k) .
\end{aligned}
$$

Then, defining $v(k):=V_{T}\left(k, \phi_{T}^{x}\left(k, k_{\circ}, x_{\circ}, \omega_{\left[k_{\circ}, k\right)}^{z}\right)\right)$, using (7), (8), the following holds along trajectories:

$$
v(k+1)-v(k) \leq-T 2 \alpha(v(k))+T\left[\nu_{1}+u(k)\right]
$$

for all $k \geq k_{\circ} \geq 0$. From this inequality we can see that the following hold true for any $k \geq k_{\circ} \geq 0$ :

$$
\begin{aligned}
v(k) & \geq \alpha^{-1}\left(\nu_{1}+\left|\omega_{\left[k_{0}, k\right)}^{z}\right|\right) \Longrightarrow v(k+1)-v(k) \\
& \leq-T \alpha(v(k)) \\
v(k+1) & \leq v(k)+T\left(\nu_{1}+\left|\omega_{\left[k_{0}, k\right)}^{z}\right|\right) .
\end{aligned}
$$

Define next $\zeta(t):=v(k)+((t / T)-k)(v(k+1)-v(k))$ for all $t \in[k T,(k+1) T), k \geq k_{\circ} \geq 0$. Notice that since $\zeta(t)$ is a linear interpolation from $v(k)$ to $v(k+1)$, which are always nonnegative, we have that

$$
\begin{aligned}
& 0 \leq \zeta(t) \leq \max \{v(k), v(k+1)\} \quad \text { for any } \\
& t \in[k T,(k+1) T), \quad k \geq k_{\circ} \geq 0 .
\end{aligned}
$$

Moreover, since trajectories of (1) and (2) are defined for all $k \geq k_{\circ} \geq 0$, the variable $\zeta(t)$ is defined for all $t \geq t_{\circ} \geq 0$. Introduce $\bar{u}(t):=u(k), \forall t \in[k T,(k+1) T), k \geq k_{\circ} \geq 0$. Using definition of $\tilde{\alpha}, T^{*}<1$, (75)-(77), we can write for all $t \in[k T,(k+1) T), k \geq k_{\circ} \geq 0$

$$
\begin{aligned}
\zeta(t) & \geq \tilde{\alpha}\left(\nu_{1}+\left|\bar{u}_{\left[t_{\circ}, t\right)}\right|\right) \\
& \Longrightarrow v(k) \geq \alpha^{-1}\left(\nu_{1}+\left|\omega_{\left[k_{\circ}, k\right)}^{z}\right|\right) \\
& \Longrightarrow v(k+1)-v(k) \leq-T \alpha(v(k)) \\
& \Longrightarrow \dot{\zeta}(t) \leq-T \alpha(v(k)) \\
& \Longrightarrow \dot{\zeta}(t) \leq-T \alpha(\zeta(t))
\end{aligned}
$$

where the last inequality follows from the fact that $v(k+1) \leq$ $v(k)$ and hence $\zeta(t) \leq v(k)$ for all $t \in[k T,(k+1) T), k \geq$ $k_{\circ} \geq 0$. Using [15, Corollaries 5.1 and 5.2], we have that

$$
\zeta(t) \leq \max \left\{\beta\left(\zeta_{\circ}, t-t_{\circ}\right), \tilde{\alpha}\left(\nu_{1}+\left|\bar{u}_{\left[t_{\circ}, t\right)}\right|\right)\right\}
$$

and using $\phi_{T}^{x}\left(k, k_{\circ}, x_{\circ}, \omega_{\left[k_{\circ}, k\right)}^{z}\right)=\zeta(k T), \bar{u}(k T)=u(k), \beta_{y}$ and $\nu_{1}$, (7) and the fact that for arbitrary $\alpha \in \mathcal{K}_{\infty}$ we have $\alpha(r+s) \leq \alpha(2 s)+\alpha(2 r), \forall r, s \geq 0$ we can write

$$
\begin{aligned}
\mid \phi_{T}^{x} & \left(k, k_{\circ}, x_{\circ}, \omega_{\left[k_{\circ}, k\right)}^{z}\right) \mid \\
\leq & \alpha_{1}^{-1}\left(\operatorname { m a x } \left\{\beta\left(\alpha_{2}\left(\left|x_{\circ}\right|\right),\left(k-k_{\circ}\right) T\right),\right.\right. \\
& \left.\left.\times \tilde{\alpha}\left(\nu_{1}+\left|\omega_{\left[k_{\circ}, k\right)}^{z}\right|\right)\right\}\right) \\
\leq & \max \left\{\alpha_{1}^{-1}\left(\beta\left(\alpha_{2}\left(\left|x_{\circ}\right|\right),\left(k-k_{\circ}\right) T\right)\right),\right. \\
& \left.\times \alpha_{1}^{-1} \circ \tilde{\alpha}\left(2 \nu_{1}\right)+\alpha_{1}^{-1} \circ \tilde{\alpha}\left(\left|\omega_{\left[k_{\circ}, k\right)}^{z}\right|\right)\right\} \\
\leq & \max \left\{\beta_{y}\left(\left|x_{\circ}\right|,\left(k-k_{\circ}\right) T\right), \nu\right\} \\
& +\gamma\left(\left|\omega_{\left[k_{\circ}, k\right)}^{z}\right|\right)
\end{aligned}
$$

for all $k \geq k_{\circ} \geq 0$.

\section{APPENDIX B}

PROOF OF PROPOSITION 2

The result is only proved for USC since the proof of UGC follows the same steps. Let $\kappa \in \mathcal{K}$ come from USB. Let $\Delta>0$ be given and let $T_{1}^{*}>0$ be generated using USB. Let $\Delta_{1}:=$ $\kappa(\Delta)+2 c$. Let $\Delta_{2}>0$ be arbitrary. Let $\left(\Delta_{1}, \Delta_{2}\right)$ generate 
$K, T_{2}^{*}>0$ using conditions of the Proposition. Let $T^{*}:=$ $\min \left\{T_{1}^{*}, T_{2}^{*}\right\}$. Consider arbitrary numbers $\eta \in(0, \Delta), \epsilon, L>0$ and define

$$
\mu:=\min \left\{\frac{\min \{\epsilon, 1\}}{e^{K L}-1}, \Delta_{2}\right\} .
$$

Consider arbitrary $\left|x_{\circ}\right| \leq \eta,\left|\omega^{z}\right| \leq \mu, T \in\left(0, T^{*}\right)$. USB implies that whenever $\left|x_{\circ}\right| \leq \Delta, T \in\left(0, T^{*}\right)$ then $\left|\phi_{T}^{x}\left(k, k_{\circ}, x_{\circ}, 0\right)\right| \leq \kappa(\Delta)+c<\Delta_{1}$ for all $k \geq k_{\circ} \geq 0$. Notice moreover that without loss of generality we may assume that $c \geq 1$. With the goal of showing contradiction suppose that there exists $k_{1}^{*} \in\left(k_{\circ}, k_{\circ}+\ell_{L, T}\right)$ such that $\left|\phi_{T}^{x}\left(k, k_{\circ}, x_{\circ}, \omega_{\left[k_{\circ}, k\right)}^{z}\right)\right| \leq \Delta_{1}$ for all $k \in\left[k_{\circ}, k_{1}^{*}\right)$ and $\left|\phi_{T}^{x}\left(k_{1}^{*}, k_{\circ}, x_{\circ}, \omega_{\left[k_{\circ}, k_{1}^{*}\right)}^{z}\right)\right|>\Delta_{1}$. Then, we can write

$$
\begin{aligned}
& \left|\phi_{T}^{x}\left(k, k_{\circ}, x_{\circ}, \omega_{\left[k_{\circ}, k\right)}^{z}\right)-\phi_{T}^{x}\left(k, k_{\circ}, x_{\circ}, 0\right)\right| \\
& \quad \leq K T \sum_{j=0}^{k-k_{\circ}-1}(1+K T)^{j}\left|\omega_{\left[k_{\circ}, k\right)}^{z}\right| \\
& \quad \leq\left[(1+K T)^{k-k_{\circ}}-1\right]\left|\omega^{z}\right| \\
& \quad \leq\left[e^{K T\left(k-k_{\circ}\right)}-1\right]\left|\omega^{z}\right| \\
& \quad \leq\left[e^{K L}-1\right]\left|\omega^{z}\right| \\
& \quad \leq \min \{\epsilon, 1\} \leq c
\end{aligned}
$$

for all $k \in\left[k_{\circ}, k_{1}^{*}\right]$. This contradicts the assumption that $\left|\phi_{T}^{x}\left(k_{1}^{*}, k_{\circ}, x_{\circ}, \omega_{\left[k_{\circ}, k_{1}^{*}\right)}^{z}\right)\right|>\Delta_{1}$ since

$$
\begin{aligned}
\mid \phi_{T}^{x} & \left(k_{1}^{*}, k_{\circ}, x_{\circ}, \omega_{\left[k_{\circ}, k_{1}^{*}\right)}^{z}\right) \mid \\
\leq & \left|\phi_{T}^{x}\left(k_{1}^{*}, k_{\circ}, x_{\circ}, 0\right)\right| \\
& +\left|\phi_{T}^{x}\left(k_{1}^{*}, k_{\circ}, x_{\circ}, \omega_{\left[k_{\circ}, k_{1}^{*}\right)}^{z}\right)-\phi_{T}^{x}\left(k_{1}^{*}, k_{\circ}, x_{\circ}, 0\right)\right| \\
\leq & \kappa(\Delta)+2 c=\Delta_{1} .
\end{aligned}
$$

Hence, (80) holds for all $k \geq k_{\circ}+\ell_{L, T}$, which completes the proof.

\section{APPENDIX C \\ PROOF OF PROPOSITION 1}

First, we show that there exist strictly positive numbers $T_{1}^{*}, \bar{c}_{1}, \bar{c}_{2}, \bar{c}_{3}, \bar{K}$ such that for all $T \in\left(0, T_{1}^{*}\right), k \geq 0$ and $x \in \mathbb{R}^{n}$ we have

$$
\begin{aligned}
-\bar{c}_{1} \psi(x) & \leq W_{T}(k, x) \leq-\bar{c}_{2} \psi(x) \\
\frac{\Delta W_{T}}{T} & \leq p_{T}(k) c_{2} \psi(x)+\bar{K}\left|\frac{\Delta V_{T}}{T}\right|-\bar{c}_{3} c_{1} \psi(x) .
\end{aligned}
$$

Let $\hat{T}_{1}>0$ come from inequalities (89) and (91). Let $\mu, L, \hat{T}_{2}$ come from the PE condition. Let $p_{M}$ come from the conditions of the proposition. Let $\hat{T}_{3}>0$ and $\hat{T}_{4}>0$ be such that

$$
\begin{array}{r}
\frac{T}{1-e^{-T}} \leq 2 \quad \forall T \in\left(0, \hat{T}_{3}\right) \quad \frac{1-e^{-T}}{T} \geq \frac{1}{2} \\
\forall T \in\left(0, \hat{T}_{4}\right) .
\end{array}
$$

Let $\hat{T}_{5}:=\left(\mu e^{-L}\right) /\left(4\left(1-e^{-L}\right)\right)$ and, finally, define $T_{1}^{*}:=$ $\min \left\{\hat{T}_{1}, \hat{T}_{2}, \hat{T}_{3}, \hat{T}_{4}, \hat{T}_{5}\right\}$. Consider arbitrary $x \in \mathbb{R}^{n}, k \geq 0$ and
$T \in\left(0, T_{1}^{*}\right)$. The first inequality in (81) holds using our choice of $\hat{T}_{3}$

$$
\begin{aligned}
T \sum_{i=k}^{\infty} e^{(k-i) T} p_{T}(i) & \leq p_{M} T \sum_{j=0}^{\infty}\left(e^{-T}\right)^{j}=p_{M} \frac{T}{1-e^{-T}} \\
& \leq 2 p_{M}=: \bar{c}_{1} .
\end{aligned}
$$

The second inequality in (81) follows from the PE condition. In particular, denote $k_{j}:=k+j \ell_{L, T}$ and since $T \ell_{L, T}=L$, we can write

$$
\begin{aligned}
T \sum_{i=k}^{\infty} e^{(k-i) T} p_{T}(i) & =\sum_{j=0}^{\infty} T \sum_{i=k+j \ell_{L}, T}^{k+(j+1) \ell_{L, T}} e^{(k-i) T} p_{T}(i) \\
& \geq \sum_{j=0}^{\infty} e^{-j T \ell_{L}, T}\left(T \sum_{i=k_{j}}^{k_{j}+\ell_{L}, T} e^{\left(k_{j}-i\right) T} p_{T}(i)\right) \\
& \geq \sum_{j=0}^{\infty} e^{-j L} e^{-\ell_{L}, T}\left(T \sum_{i=k_{j}}^{k_{j}+\ell_{L}, T} p_{T}(i)\right) \\
& \geq \frac{e^{-L}}{1-e^{-L}} \mu=: \bar{c}_{2} .
\end{aligned}
$$

We show next that (82) holds. Using similar calculations as in (84), (86), and our choice of $\hat{T}_{4}$ and $\hat{T}_{5}$, we have

$$
\begin{aligned}
& \frac{W_{T}\left(k+1, F_{T}\right)-W_{T}(k, x)}{T} \\
&=-\sum_{i=k+1}^{\infty} e^{(k+1-i) T} p_{T}(i) V_{T}\left(k+1, F_{T}\right) \\
& \quad+\sum_{i=k}^{\infty} e^{(k-i) T} p_{T}(i) V_{T}(k, x) \\
&=p_{T}(k) V_{T}(k, x)-\sum_{i=k+1}^{\infty} e^{(k+1-i) T} p_{T}(i) \\
& \quad \times\left[V_{T}\left(k+1, F_{T}\right)-V_{T}(k, x)\right] \\
& \quad-\left(1-e^{-T}\right) \sum_{i=k+1}^{\infty} e^{(k+1-i) T} p_{T}(i) V_{T}(k, x) \\
& \leq p_{T}(k) V_{T}(k, x)+\left(p_{M} T \sum_{j=0}^{\infty} e^{-j T}\right)\left|\frac{\Delta V_{T}}{T}\right| \\
& \quad-\frac{1-e^{-T}}{T} T \sum_{i=k+1}^{\infty} e^{(k+1-i) T} p_{T}(i) V_{T}(k, x) \\
& \leq p_{T}(k) V_{T}(k, x)+\frac{p_{M} T}{1-e^{-T}}\left|\frac{\Delta V_{T}}{T}\right|-\frac{\bar{c}_{2}}{2} V_{T}(k, x)
\end{aligned}
$$

where for the last term we used (83) and (86). This completes the proof of (81) and (82) with $\bar{c}_{3}=\left(\bar{c}_{2}\right) /(2)$ and $\bar{K}=\left(p_{M} T_{1}^{*}\right) /\left(1-e^{-T_{1}^{*}}\right)$. Next, combining (18) and (87) we have that

$$
\begin{aligned}
\frac{\Delta U_{T} \leq}{T} \leq & -c_{3}\left|p_{T}\right| \psi+T c_{4} \psi+\varepsilon\left|p_{T}\right| c_{2} \psi+\varepsilon \bar{K} c_{3}\left|p_{T}\right| \psi \\
& +\varepsilon^{2} \frac{\bar{K} \bar{c}_{3} c_{1}}{2} \psi-\varepsilon \frac{\bar{c}_{2} c_{1}}{4} \psi-\varepsilon \frac{\bar{c}_{3} c_{1}}{4} \psi-\varepsilon \frac{\bar{c}_{3} c_{1}}{4} \psi
\end{aligned}
$$


from which it is easy to see that, defining $\epsilon^{*}:=$ $\min \left\{\left(c_{1}\right) /\left(2 \bar{c}_{1}\right),(1) /(2 \bar{K}),\left(c_{2}\right) /\left(2 \bar{c}_{2}\right),\left(c_{3}\right) /\left(c_{2}+\bar{K} c_{3}\right)\right\}$ and $T^{*}:=\min \left\{T_{1}^{*},\left(\epsilon \bar{c}_{3} c_{1}\right) /\left(2 c_{4}\right)\right\}$, we obtain that $(21),(22)$ hold with $d_{1}=\left(c_{1} / 2\right), d_{2}=\left(c_{2} / 2\right)$ and $d_{3}=\left(\epsilon \bar{c}_{3} c_{1}\right) /(4)$, which completes the proof.

\section{APPENDIX D \\ PROOF OF PROPOSITION 4}

It is worth recalling to avoid confusion in the notation, that the state $X$ in Proposition 3 corresponds here to $X=\operatorname{col}\left[x_{e}, y_{e}\right]$ and the input $z$ in Proposition 3 corresponds here to $\theta_{e}$. We use Theorem 2 to prove this result ${ }^{2}$. First, we see that Assumption 1 holds trivially in view of item 1 ) of Proposition 4 . To see more clearly, notice that (37) implies that $f_{T}(\cdot, \cdot, \cdot)$ is continuous and uniformly bounded in the first argument. Item 1) of Proposition 4 also implies that there exists $c>0$ independent of $T$, such that for all $T \in\left(0, T^{*}\right)$, we have that $\left|G_{T}(k, X, z)\right| \leq T c|z|(|X|+$ 1).

Second, it is evident that the origin of (36) is uniformly globally exponentially stable for any $a_{1}$ and any $T \in\left(0, T^{*}\right)$ where $T^{*}>0$ is such that $1>a_{1} T^{*}>0$ and therefore the trajectories $\phi_{T}^{z}(\cdot, \cdot, \cdot)$ are uniformly summable.

It is left to prove that the unperturbed dynamics $X(k+1)=$ $F_{1 T}(k, X(k))$ in (35) is UGAS and that (35) is UGB. As a matter of fact we will show that the zero-input system in (35) is Lyapunov UGES.

Proof of UGES of $X(k+1)=F_{1 T}(k, X(k))$ : We use Proposition 1. To that end, consider the function $V_{T}(k, X):=$ $|X|^{2}-\varepsilon \omega_{r_{k-1}} x_{e} y_{e}$ with $\varepsilon:=\alpha_{y}+T$ and $\alpha_{y}>0$. Observe that this function is positive definite and radially unbounded for sufficiently small $\alpha_{y}, T^{*}$ and $w_{M}$; indeed, we have that

$$
c_{1}|X|^{2} \leq V_{T}(k, X) \leq c_{2}|X|^{2}
$$

with $c_{1}:=\left(1-0.5\left(\alpha_{y}+T^{*}\right) w_{M}\right)$ and $c_{2}:=\left(1+0.5\left(\alpha_{y}+\right.\right.$ $\left.\left.T^{*}\right) w_{M}\right)$ which are clearly independent of $T$. We assume that $\alpha$ and $T^{*}$ are sufficiently small so that $c_{1}>0$. So, (17) holds with $\psi(X)=|X|^{2}$. Next, we compute

$$
\begin{aligned}
\Delta V_{T}:= & V_{T}\left(k, F_{1 T}(k, X)\right)-V_{T}(k, X) \\
= & -T\left(2 a_{2}-\varepsilon \omega_{r_{k}}^{2}\right) x_{e}^{2}-\varepsilon T \omega_{r_{k}}^{2} y_{e}^{2} \\
& +T^{2} x_{e}\left(\left[a_{2}^{2}+\omega_{r_{k}}^{2}-\varepsilon a_{2}\right] x_{e}\right. \\
& \left.-\left[2 a_{2} \omega_{r_{k}}-\varepsilon \omega_{r_{k}}^{3}\right] y_{e}-\left[2\left(1-a_{2} T\right)+\varepsilon \omega_{r_{k}}^{2} T\right] \vartheta\right) \\
& +T^{2} y_{e}\left(\omega_{r_{k}}^{2} y_{e}+\varepsilon \omega_{r_{k}} \vartheta\right)+T^{4} \vartheta^{2} \\
& +\varepsilon x_{e} y_{e}\left(\omega_{r_{k}}-\omega_{r_{k-1}}+\omega_{r_{k}} a_{2} T\right) .
\end{aligned}
$$

Under the assumptions of the proposition, $\varepsilon x_{e} y_{e}\left(\omega_{r_{k}}-\right.$ $\left.\omega_{r_{k-1}}+\omega_{r_{k}} a_{2} T\right) \leq(1 / 2)\left[T^{2} y_{e}^{2}+\varepsilon^{2} w_{M}^{2}\left(1+a_{2}\right)^{2} x_{e}^{2}\right]$. Define $\alpha_{x}:=a_{2}-\left(\alpha_{y}+T^{*}\right) w_{M}^{2}-(1 / 2) \varepsilon^{2} w_{M}^{2}\left(1+a_{2}\right)^{2}$ which is positive for sufficiently small values of $\varepsilon$ and sufficiently

\footnotetext{
${ }^{2}$ We could also use Theorem 1 . Notice that to show that the system (1) is UGC with input $\theta_{e}$ we may appeal to Lemma 2 observing that the system $X(k+1)=$ $f_{T}\left(k, X(k), \theta_{e}(k)\right)$ with $f_{T}\left(k, X, \theta_{e}\right):=F_{1 T}(k, X)+G_{1 T}\left(k, X, \theta_{e}\right)$ is linear in the state $X=\operatorname{col}\left[x_{e}, y_{e}\right]$ and for each $\Delta>0$, we have that $f_{T}\left(k, X, \theta_{e}\right)$ is globally Lipschitz in $\theta_{e}$ uniformly for all $k \geq 0$ and $X$ such that $|X| \leq \Delta$, with a Lipschitz constant of the form $L=L^{\prime} T$ with $L^{\prime}$ depending only on $\Delta$.
}

large values of $a_{2}$. Also, since $|\vartheta(k, X)| \leq K|X|$ there exists $K_{1}>0$ such that

$$
\begin{aligned}
\frac{\Delta V_{T}}{T} & \leq-\left(\alpha_{x} x_{e}^{2}+\alpha_{y} \omega_{r_{k}}^{2} y_{e}^{2}\right)+T K_{1}|X|^{2} \\
& \leq-p_{T}(k)|X|^{2}+T K_{1}|X|^{2}
\end{aligned}
$$

for all $T \in\left(0, T^{*}\right), X \in \mathbb{R}^{2}$ and $k \geq 0$. Hence, (18) holds with $c_{4}=K_{1}, c_{3}=1$ and $p_{T}(k):=\min \left\{\alpha_{x}, \alpha_{y} \omega_{r_{k}}^{2}\right\}$. Notice that (19) also holds in view of (37). Finally, $p_{T}(\cdot)$ is PE since $\omega_{r}^{2}(\cdot)$. We conclude that there exists a function $U_{T}: \mathbb{Z}_{\geq 0} \times$ $\mathbb{R}^{2} \rightarrow \mathbb{R}$ such that (21) and (22) hold. Exponential convergence is concluded from the fact that $\psi(X)=|X|^{2}$.

Proof of $U G B$ : We invoke Proposition 1. We proceed to verify the conditions of Proposition 3 with $f_{T}(k, X, z):=F_{1 T}(k, X)+G_{T}(k, X, z)$ as defined in (35) and with $V_{T}(k, X)=U_{T}(k, X)$. Bounds (25) and (26) hold in view of (21) and (22). Conditions (26) and (27) hold with $\varphi(s)=s, \tilde{\gamma}_{2}=\kappa_{2} s$, and $\tilde{\gamma}_{1}(s):=\kappa_{3} s, \kappa_{2}, \kappa_{3}>0$. This is because $U_{T}(k, \cdot)$ is bounded from above and below by quadratic terms and $G_{T}(k, X, z)$ is of linear growth in $X$ for each fixed $k$ and $z$ and trigonometric functions, which can be over-bounded by a linear function of $X$. Also, $G_{T}(k, X, \cdot)$ can be over-bounded by a linear function for each fixed $k$ and $X$. Finally, (28) holds with a linear function $\rho(s):=\rho s$ since $\mu(s)$ is in this case a nondecreasing function of linear growth and $\phi_{T}^{\theta_{e}}(k)$ decays uniformly exponentially to zero.

\section{REFERENCES}

[1] P. Albertos, "Sampled-data modeling and control of nonlinear systems," in Proc. 35th IEEE Conf. Decision Control, Kobe, Japan, 1996, pp. 925-930.

[2] D. Angeli, "Intrinsic robustness of global asymptotic stability," Syst. Control Lett., vol. 38, pp. 297-307, 1999.

[3] M. Arcak, D. Angeli, and E. Sontag, "A unifying integral ISS framework for stability of nonlinear cascades," SIAM J. Control Optim., vol. 40, pp. 888-1904, 2002.

[4] M. S. Branicky, "Stability of switched and hybrid systems," in Proc. 33rd. IEEE Conf. Decision Control, Orlando, FL, 1994, pp. 3498-3503.

[5] D. Dochain and G. Bastin, "Adaptive identification and control algorithms for nonlinear bacterial growth systems," Automatica, vol. 20, pp. 621-634, 1984.

[6] G. C. Goodwin, B. McInnis, and R. S. Long, "Adaptive control algorithm for waste water treatment and $\mathrm{pH}$ neutralization," Optimal Control Applicat. Meth., vol. 3, pp. 443-459, 1982.

[7] L. Grüne and D. Nešić, "Optimization based stabilization of sampled-data nonlinear systems via their approximate discrete-time models," SIAM J. Control Optim., vol. 42, no. 1, pp. 98-122, 2003.

[8] L. Hou, A. N. Michel, and Y. Hui, "Some qualitative properties of sampled-data control systems," in Proc. 35th IEEE Conf. Decision Control, Kobe, Japan, 1996, pp. 911-916.

[9] "Special Issue on Hybrid Control Systems," IEEE Trans. Automat. Contr., vol. 43, Apr. 1998.

[10] M. Janković, R. Sepulchre, and P. V. Kokotović, "Constructive Lyapunov stabilization of nonlinear cascaded systems," IEEE Trans. Automat. Contr., vol. 41, pp. 1723-1736, Oct. 1996.

[11] Z. P. Jiang, "Iterative design of time-varying stabilizers for multi-input systems in chained form," Syst. Contr. Lett., vol. 28, pp. 255-262, 1996.

[12] Z.-P. Jiang and Y. Wang, "Input-to-state stability for discrete-time nonlinear systems," Automatica, vol. 37, no. 6, pp. 857-869, 2001.

[13] Y. Kanayama, Y. Kimura, F. Miyazaki, and T. Naguchi, "A stable traking control scheme for an autonomous vehicle," in Proc. IEEE Conf. Robotics Automation, 1990, pp. 384-389.

[14] C. Kellett, "Advances in converse and control Lyapunov function," Ph.D. dissertation, Univ. California, Santa Barbara, CA, June 2002.

[15] H. Khalil, Nonlinear Systems, 2nd ed. New York: Macmillan, 1996.

[16] I. Kolmanovsky and H. McClamroch, "Developments in nonholonomic control problems," Control Syst. Mag., pp. 20-36, Dec. 1995. 
[17] S. Kotsios, "A new factorization of special nonlinear discrete systemsand their applications," IEEE Trans. Automat. Contr., vol. 45, pp. 24-33, Jan. 2000.

[18] U. Kotta, Inversion Method in Discrete-Time Nonlinear Control Systems Synthesis Problems. London, U.K.: Springer-Verlag, 1995.

[19] D. S. Laila and D. Nešić, "Changing supply rates for input-output to state stable discrete-time nonlinear systems with applications," Automatica, vol. 39, no. 5, pp. 821-835, 2003

[20] A. A. J. Lefeber, "Tracking control of nonlinear mechanical systems," Ph.D. dissertation, Univ. Twente, Enschede, The Netherlands, 2000.

[21] D. Liberzon and A. S. Morse, "Basic problems in stability and design of switched systems," IEEE Control Syst. Mag., pp. 59-70, Oct. 1999.

[22] A. Loría. (2001, Nov.) Cascaded nonlinear time-varying systems: Analysis and design, 2000, Mexico CIty, Mexico. [Online]. Available: http://public.lss.supelec.fr/perso/loria/Teaching/course-casc-ipn.html

[23] A. Loría and D. Nešić, "On uniform boundedness of parameterized discrete-time systems with decaying inputs: Applications to cascades," Syst. Control Lett., vol. 49, no. 3, pp. 163-174, 2003.

[24] A. Loría, E. Panteley, D. Popović, and A. Teel, "An extension of Matrosov's theorem with application to nonholonomic control systems," in Proc. 40th IEEE Conf. Decision Control, Las Vegas, NV, 2002, Paper no. REG0625.

[25] I. M. Y. Mareels, H. B. Penfold, and R. J. Evans, "Controlling nonlinear time-varying systems via Euler approximations," Automatica, pp. 681-696, 1992.

[26] F. Mazenc, "Strict Lyapunov functions for time-varying systems," $\mathrm{Au}$ tomatica, vol. 39, pp. 349-353, 2003.

[27] F. Mazenc and L. Praly, "Adding integrators, saturated controls and global asymptotic stabilization of feedforward systems," IEEE Trans. Automat. Contr., vol. 41, pp. 1559-1579, Nov. 1996.

[28] S. Monaco and D. Normand-Cyrot, "On the sampling of a linear analytic control system," in Proc. 24th IEEE Conf. Decision Control, Fort Lauderdale, FL, 1985, pp. 1457-1462.

[29] - On Nonlinear Digital Control, Volume 3 of Control Chapter in Nonlinear Systems, A. J. Fossard and D. Normand-Cyrot, Eds. New York: Chapman \& Hall, 1995.

[30] P. Morin and C. Samson, "A characterization of the Lie algebra rank condition by transverse periodic functions," in SIAM J. Control Optim. vol. 40, 2002, pp. 1227-1249.

[31] D. Nešić and A. Loría. (2003, July) On uniform asymptotic stability of time-varying parameterized discrete-time cascades. [Online]. Available: http://front.math.ucdavis.edu/math.OC/0 307167

[32] D. Nešić and A. Loría, "Uniform practical asymptotic stability of timevarying parameterized discrete-time cascades," presented at the 41st. IEEE Conf. Decision Contr., Honolulu, HI, 2003, Paper no. 587.

[33] D. Nešić and A. Teel, "Matrosov theorem for parameterized families of discrete-time systems," Automatica, vol. 40, no. 6, pp. 1025-1034, 2003

[34] D. Nešić, A. Teel, and P. Kokotović, "Sufficient conditions for stabilization of sampled-data nonlinear systems via discrete-time approximations," Syst. Control Lett., vol. 38, pp. 259-270, 1999

[35] D. Nešić, A. Teel, and E. Sontag, "Formulas relating $\mathcal{K} \mathcal{L}$ stability estimates of discrete-time and sampled-data nonlinear systems," Syst. Control Lett., vol. 38, pp. 49-60, 1999.

[36] D. Nešić and A. R. Teel, "A framework for stabilization of nonlinear sampled-data systems based on their approximate discrete-time models," IEEE Trans. Automat. Contr., 2004, to be published.

[37] E. Panteley, E. Lefeber, A. Loría, and H. Nijmeijer, "Exponential tracking of a mobile car using a cascaded approach," in Proc. IFAC Workshop Motion Control, Grenoble, France, 1998, pp. 221-226.

[38] E. Panteley and A. Loría, "On global uniform asymptotic stability of non linear time-varying non autonomous systems in cascade," Syst. Control Lett., vol. 33, no. 2, pp. 131-138, 1998.

[39] _ - "Growth rate conditions for stability of cascaded time-varying systems," Automatica, vol. 37, no. 3, pp. 453-460, 2001.

[40] C. Rui, I. Kolmanovsky, and N. H. McClamroch, "Hybrid control for stabilization of cascade nonlinear systems," in Proc. Amer. Control Conf., 1997, pp. 2800-2804.

[41] A. Saberi, P. V. Kokotović, and H. J. Sussmann, "Global stabilization of partially linear systems," SIAM J. Control Optim., vol. 28, pp. $1491-1503,1990$.
[42] G. Sansone and R. Conti, Nonlinear Differential Equations, Oxford, U.K.: Pergamon, 1964

[43] E. D. Sontag, "Remarks on stabilization and input-to-state stability," in Proc. 28th. IEEE Conf. Decision Control, Tampa, Fl, 1989, pp. 1376-1378.

[44] — "Comments on integral variants of ISS," Syst. Control Lett., vol. 34, pp. 93-100, 1998

[45] E. D. Sontag and Y. Wang, "On characterizations of the input-to-state stability property," Syst. Control Lett., vol. 24, pp. 351-359, 1995

[46] J. A. Stiver, P. J. Antsaklis, and M. D. Lemmon, "Digital control from a hybrid perspective," in Proc. 33rd IEEE Conf. Decision Control, Orlando, FL, 1994, pp. 4241-4246.

[47] A. M. Stuart and A. R. Humphries, Dynamical Systems and Numerical Methods. Cambridge, U.K.: Cambridge Univ. Press, 1996.

[48] H. J. Sussman and P. V. Kokotović, "The peaking phenomenon and the global stabilization of nonlinear systems," IEEE Trans. Automat. Contr. vol. 36, pp. 424-439, Apr. 1991.

[49] J. Tsinias, "Stabilizability of discrete-time systems," IMA J. Math. Control Infrom., no. 6, pp. 135-150, 1989.

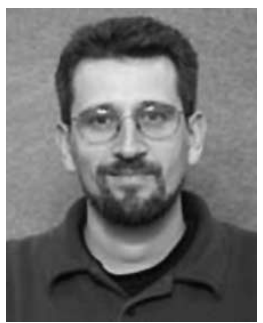

Dragan Nesic received the B.M.E. degree from the University of Belgrade, Belgrade, Yugoslavia and the Ph.D. degree in systems engineering from the Australian National University, Canberra, in 1990 and 1997, respectively.

He is currently an Associate Professor and Reader in the Department of Electrical and Electronic Engineering, the University of Melbourne, Melbourne, Australia. He is currently serving as a Deputy Head of the Department. From 1997 to 1999, he held several postdoctoral positions: DEEE, The University of Melbourne, Australia; CESAME, Universite Catholique de Louvain, Louvain la Neuve, Belgium; and ECE, University of California at Santa Barbara. Since February 1999, he has been with The University of Melbourne. He is an Associate Editor for the journals Automatica and Systems and Control Letters, and Associate Editor of the Conference Editorial Board for The American Control Conference and The IEEE Conference on Decision and Control.

Dr. Nesic was awarded a postodoctoral fellowship to carry out his research in Belgium under the Inter-University Poles of Attraction programme funded by the Belgian Government, in 1997. He was recently awarded a Humboldt Research Fellowship by the Alexander von Humboldt Foundation (Germany) in order to pursue research in Germany for a period of six months. He is a Senior Member of IEAust.

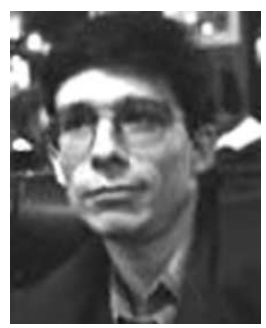

Antonio Loría (SM'91-M'96) was born in Mexico in 1969. He received the B.Sc. degree in electronic engineering from the ITESM, Monterrey, Mexico, in 1991, and the M.Sc. and Ph.D. degrees in control engineering from the UTC, Paris, France, in 1993 and 1996, respectively.

From December 1996 through December 1998 he was an Associate Researcher at the University of Twente, Enschede, The Netherlands; NTNU, Norway, and the University of California at Santa Barbara. Since 1999, he has been "Charge de Recherche" at the the French National Centre of Scientific Research (CNRS). He has been with the "Laboratoire de Signaux et Systemes" Gif sur Yvette, France, since December 2002. He is (co)author of about 80 scientific articles and the book Passivity Based Control of Euler-Lagrange Systems (New York: Springer-Verlag, 1998). His research interests include: Modeling and control of Euler-Lagrange systems, stability analysis of nonlinear time-varying systems, biped locomotion, and output feedback stabilization. Dr. Loria is an Associate Editor of Systems and Control Letters. Detailed information and publications are availble at http://public.lss.supelec.fr/perso/loria. 